\title{
Incidence and reflection of internal waves and wave-induced currents at a jump in buoyancy frequency
}

\author{
J. P. McHugh \\ University of New Hampshire, Durham, NH, USA \\ Correspondence to: J. P. McHugh (john.mchugh@unh.edu)
}

Received: 13 February 2014 - Published in Nonlin. Processes Geophys. Discuss.: 21 March 2014

Revised: 17 March 2015 - Accepted: 28 March 2015 - Published: 4 May 2015

\begin{abstract}
Weakly nonlinear internal gravity waves are treated in a two-layer fluid with a set of nonlinear Schrodinger equations. The layers have a sharp interface with a jump in buoyancy frequency approximately modeling the tropopause. The waves are periodic in the horizontal but modulated in the vertical and Boussinesq flow is assumed. The equation governing the incident wave packet is directly coupled to the equation for the reflected packet, while the equation governing transmitted waves is only coupled at the interface. Solutions are obtained numerically. The results indicate that the waves create a mean flow that is strong near and underneath the interface, and discontinuous at the interface. Furthermore, the mean flow has an oscillatory component that can contaminate the wave envelope and has a vertical wavelength that decreases as the wave packet interacts with the interface.
\end{abstract}

\section{Introduction}

Earth's tropopause often has a vertical structure with a very sudden change in the lapse rate with increasing altitude, and a corresponding sudden increase in the buoyancy frequency $N$. This sudden increase in $N$ can restrict upwardly propagating internal waves, as has been known for some time (Scorer, 1949). Observations of flow in the vicinity of the tropopause have also shown unusual dynamic behavior including high turbulence levels (Partl, 1962; Worthington, 1998; Wolff and Sharman, 2008; McHugh et al., 2008b) and large wave amplitudes (McHugh et al., 2008a; Smith et al., 2008). Turbulence and waves at these altitudes are important aspects of weather and climate, and serious hazards to aircraft.
McHugh (2008, 2009) considered horizontally periodic internal waves interacting with an idealized model of the tropopause. The waves in McHugh (2009) were uniform while the waves in McHugh (2008) were confined to a vertical packet and treated with numerical simulations. The results indicate that while nonlinear effects are stronger near the interface even with uniform waves, a modulated amplitude results in a localized jet-like mean flow near the interface that can be strong enough to form a critical layer, with important consequences for later waves.

Recently Grimshaw and McHugh (2013) treated weakly nonlinear two-layer horizontally periodic waves for both unsteady and steady flow. For unsteady flow, they show expressions for the wave-induced mean flow for large time. They then use these expressions to treat the steady problem. They did not determine the evolution of the wave packet while it transits the interface, which is treated here.

A related configuration is a layer with constant $N$ (no interface), treated theoretically by Grimshaw (1975), Shrira (1981), Voronovich (1982), Sutherland (2006) and Tabaei and Akylas (2007). Grimshaw (1975) considered waves with a background shear flow using the wave action equation. Shrira (1981) treated weakly nonlinear waves in three dimensions assuming the modulation is the same in all directions. Voronovich (1982) also treats weakly nonlinear waves but restricts attention to the special case with the modulation along a fixed direction. The weakly nonlinear waves in Sutherland (2006) are horizontally periodic with the modulation only in the vertical. Tabaei and Akylas (2007) treat weakly nonlinear and finite amplitude theory with several different configurations for the modulation. Associated numerical simulations with constant $N$ were performed by Sutherland (2001) in two dimensions. 
Packets of internal waves that propagate at a steep angle to the horizontal will experience a modulational instability, as discussed by Sutherland (2001). Wave packets experiencing this instability will focus wave energy. Waves propagating at a shallow angle will defocus. For the waves treated here, this modulational instability may be important, depending on the wavenumbers and on the distance from the wave source to the interface. However for intermediate propagation angles, the modulational instability is too slow, and the waves interact with the interface before experiencing any significant effects.

The stability of plane monochromatic internal waves propagating at an angle to the horizontal was treated by Shrira (1981) and Tabaei and Akylas (2007), who showed that nonlinearity can lead to instability. Indeed Tabaei and Akylas (2007) show that the incident waves treated here are unstable for shallow angles. However the growth rate of the instability is second order in the nonlinearity parameter, and hence the waves treated here will interact with the interface before this instability has time to grow significantly.

The incident waves treated here are partially reflected at the interface, resulting in incident, reflected, and transmitted wave packets that are governed by coupled nonlinear Schrodinger (NLS) equations. Similar coupled NLS equations have been treated previously by Knobloch and Gibbon (1991) and Griffiths et al. (2006) starting with model equations such as the Klein-Gordon equation and groups of arbitrarily defined incident waves. The amplitude equations are similar to the equations given below.

The reflection of nonlinear internal waves by a sloping bottom has been treated by several authors; for example Thorpe (1987) treats uniform wave trains and Tabaei et al. (2005) treat wave beams. The results show that the first few higher harmonics reflect at different angles than the primary harmonic while higher harmonics are evanescent, similar to waves reflecting from the interface treated in McHugh (2009). The mean flow in Thorpe (1987) has an oscillatory component parallel to the slope with wavenumber equal to the difference between incident and reflected wavenumbers, and this is also found here. The mean flow in Tabaei et al. (2005) is more complex as they considered wave beams, and they do not report an oscillatory component; however, it is likely present in their calculations. Thorpe (1987) and Tabaei et al. (2005) do not include a modulation in the wave amplitude, and their results do not have the associated mean flow. The mean flow that is present in Tabaei et al. (2005) is confined to the region where incident and reflected waves overlap. This same feature is true here only for the oscillatory part of the mean flow. Results for a rigid horizontal lid are given here at the end for comparison and show that the interface and the rigid lid have similar behavior. The rigid boundary creates a stronger mean flow due to the stronger reflected waves.

The results given below show that the incident and reflected waves combine for a short period to create a strong localized mean flow under the interface that is discontinuous at the interface, as in Grimshaw and McHugh (2013). Furthermore there is an oscillatory component of the mean flow with a vertical wavenumber that increases as the wave packet interacts with the interface. Section 2 provides the basic equations and interfacial boundary conditions. Section 3 chooses the wave modes to be included. Section 4 discusses the important mean flow, and then Sect. 5 determines the amplitude equations. Results are discussed in Sect. 6, followed by conclusions.

\section{Basic equations}

The flow is treated as incompressible and inviscid, and attention is restricted to two dimensions. The stratification is due to the presence of a non-diffusing quantity, and the flow is assumed to be Boussinesq. The flow is then governed by

$$
\begin{aligned}
& \frac{\mathrm{D} u}{\mathrm{D} t}=-\frac{1}{\rho_{0}} \frac{\partial p}{\partial x}, \\
& \frac{\mathrm{D} w}{\mathrm{D} t}=-\frac{1}{\rho_{0}} \frac{\partial p}{\partial z}-b, \\
& \frac{\partial u}{\partial x}+\frac{\partial w}{\partial z}=0, \\
& \frac{\mathrm{D} b}{\mathrm{D} t}-N^{2} w=0,
\end{aligned}
$$

where

$$
\frac{\mathrm{D}}{\mathrm{D} t}=\frac{\partial}{\partial t}+u \frac{\partial}{\partial x}+w \frac{\partial}{\partial z},
$$

the velocity is $(u, w)$, the dynamic pressure is $p, \rho_{0}$ is an average (constant) density, and $b$ is the buoyancy, defined by

$b=\frac{g(\rho-\tilde{\rho})}{\rho_{0}}$,

where $\widetilde{\rho}$ is the background density, and $N$ is the buoyancy frequency profile, defined by

$N^{2}=-\frac{g \widetilde{\rho}_{z}}{\rho_{0}}$.

The base state must satisfy the equation of static equilibrium,

$$
\frac{\partial \widetilde{p}}{\partial z}=-\widetilde{\rho} g,
$$

where $\widetilde{p}$ is the background pressure. These equations are valid for a general profile $N(z)$.

The kinematic condition at a sharp interface between two layers is

$\eta_{t}+u \eta_{x}=w$

which holds on the interface $z=\eta$, where $\eta$ is the vertical displacement of the interface. Expand in a Taylor series in the same manner usually used for free surface flow: 


$$
\begin{aligned}
\eta_{t} & +\left[u+u_{z} \eta+\frac{1}{2} u_{z z} \eta^{2}+\cdots\right] \eta_{x} \\
& =\left[w+w_{z} \eta+\frac{1}{2} w_{z z} \eta^{2}+\cdots\right],
\end{aligned}
$$

now on $z=0$.

The dynamic condition is continuity of total pressure $p_{\mathrm{T}}$ across the interface. The total pressure is segmented into two parts:

$p_{\mathrm{T}}=\widetilde{p}+p$.

As the pressure difference along the interface is zero, the directional derivative of the pressure difference along this interface is also zero. This directional derivative may be written as

$$
\frac{\partial}{\partial x}+\eta_{x} \frac{\partial}{\partial z},
$$

which results in

$$
\left[\left(\frac{\partial}{\partial x}+\eta_{x} \frac{\partial}{\partial z}\right) p+\eta_{x} \widetilde{p}_{z}\right]_{-}^{+}=0
$$

on $z=\eta$. The equations of motion may now be used to eliminate the derivatives of the dynamic pressure, giving

$$
\begin{aligned}
& {\left[\left(u_{t}+(u u)_{x}+(w u)_{z}\right)\right.} \\
& \left.+\eta_{x}\left(w_{t}+(u w)_{x}+(w w)_{z}+b\right)-\eta_{x} \frac{1}{\rho_{0}} \widetilde{p}_{z}\right]_{-}^{+}=0
\end{aligned}
$$

on $z=\eta$. A Taylor series is used as before, leading to

$$
\begin{aligned}
& \left(1+\eta \frac{\partial}{\partial z}+\frac{\eta^{2}}{2 !} \frac{\partial^{2}}{\partial z^{2}}+\cdots\right)\left[\left(u_{t}+(u u)_{x}+(w u)_{z}\right)\right. \\
& \left.+\eta_{x}\left(w_{t}+(u w)_{x}+(w w)_{z}+b\right)\right]_{-}^{+} \\
& -\eta_{x}\left[\eta N^{2}+\frac{\eta^{2}}{2 !} \frac{\mathrm{d}}{\mathrm{d} z}\left(N^{2}\right)+\frac{\eta^{3}}{3 !} \frac{\mathrm{d}^{2}}{\mathrm{~d} z^{2}}\left(N^{2}\right)+\cdots\right]_{-}^{+}=0
\end{aligned}
$$

on $z=0$. The $N(z)$ profile considered below is a piecewise continuous profile with lower and upper layers having constant buoyancy frequency $N_{1}$ and $N_{2}$, respectively. For this profile, derivatives of $N^{2}$ in Eq. (14) are zero.

\section{A vertically modulated wavetrain}

The waves are horizontally periodic but modulated vertically. The modulation is assumed to be slow as measured by the small parameter $\epsilon$. The amplitude of the waves is also assumed small as measured by the small parameter $\alpha$. The two small parameters are assumed to have the same order so that dispersion and nonlinearity are approximately in balance. The scaling here is similar to that of Grimshaw (1975), except here the modulation is vertical and Grimshaw treated a horizontal modulation. The configuration here is the same as in Sutherland (2006), except now there are two layers. The derivation of the amplitude equations is similar to that of Shrira (1981) in that all terms are retained up to and including the order where nonlinear effects first appear, although Shrira (1981) included horizontal as well as vertical modulations. Define the following variables:

$\xi=x-c_{\mathrm{p}} t$,

$z_{j}=\epsilon^{j} z$

$t_{j}=\epsilon^{j} t$,

where $c_{\mathrm{p}}$ is the horizontal phase speed. Only $j=1,2$ are needed for the present theory.

The linear solution for a wave with upward group velocity is

$w=\alpha I\left(t_{j}, z_{j}\right) e^{i\left(k \xi-n_{1} z\right)}+\mathrm{cc}$,

where $k$ and $n_{1}$ are the horizontal and vertical wavenumbers, respectively, and cc means complex conjugate. All wavenumbers are assumed positive and $I=O(1)$. The dispersion relation is

$c_{\mathrm{p}}^{2}=\frac{N_{1}^{2}}{k^{2}+n_{1}^{2}}$.

When an interface is included, the solution in the lower layer requires the addition of reflected waves:

$\alpha R\left(t_{j}, z_{j}\right) e^{i\left(k \xi+n_{1} z\right)}+\mathrm{cc}$.

A peculiar fact of the Boussinesq equations is that when $N$ is constant in an infinite fluid (no interface), the linear purely sinusoidal solution is also a fully nonlinear solution. This can be verified by direct substitution of the periodic solution to see that the nonlinear terms for each equation are exactly zero. This fact is discussed by Thorpe (1968) and Grimshaw (1976). Tabaei and Akylas (2007) made extensive use of this fact to develop their finite amplitude theory. When the waves are contained within a slowly varying packet, then they are no longer purely sinusoidal, and nonlinear effects such as the presence of higher harmonics are expected with constant $N$ (no interface) only as a result of the modulation. These harmonics are $O\left(\epsilon \alpha^{2}\right)$ and can be made weaker by choosing a slower modulation. When incorporated into the amplitude equations, the effect of these harmonics appears at $O\left(\epsilon \alpha^{3}\right)$ and hence the higher harmonics driven by the modulation are higher order and need not be included.

Further higher harmonics are generated by nonlinear effects at the interface, as shown previously by McHugh (2009). The modulation of the wavetrain does not exert the dominant influence on these interfacial harmonics, and in fact they occur even when the wave amplitude is constant (no wave packet). Hence these interfacial harmonics cannot 
be weakened by a slow modulation of the wave packet. However the vertical wavenumber of the interfacial harmonics is not commensurate with the primary harmonic. Hence these harmonics caused by the interface do not contribute to the evolution of the primary waves and also need not be included, except at the interface where nonlinear effects may influence the reflection and transmission coefficients.

Combining all leading order contributions results in

$w=\alpha I e^{i\left(k \xi-n_{1} z\right)}+\alpha R e^{i\left(k \xi+n_{1} z\right)}+\mathrm{cc}, \quad z<0$.

The corresponding solution for the upper layer is

$w=\alpha T e^{i\left(k \xi-n_{2} z\right)}+\mathrm{cc}, \quad z>0$.

The vertical wavenumber in the upper layer is $n_{2}$, determined to first order by choosing the same wavenumber $k$ and frequency $\sigma$ for the two layers:

$\sigma=\frac{k N_{1}}{\sqrt{k^{2}+n_{1}^{2}}}=\frac{k N_{2}}{\sqrt{k^{2}+n_{2}^{2}}}$.

The linear interfacial conditions result in a relationship between the amplitudes of the incident, reflected, and transmitted wave packets at the interface:

$R=\mathcal{R} I$,

$T=\mathcal{T} I$,

on $z=0$, where

$\mathcal{R}=\frac{n_{1}-n_{2}}{n_{1}+n_{2}}$,

$\mathcal{T}=\frac{2 n_{1}}{n_{1}+n_{2}}$,

which are the reflection and transmission coefficients, respectively. These are the same coefficients that govern the reflection of light waves (see for example the text of Born and Wolf (1959) for a general discussion).

\section{The mean flow}

Separate all dynamic fields into a $\xi$-averaged mean and a fluctuating part:

$(u, w, p, b)=\left(\alpha^{2} \bar{u}+\alpha \hat{u}, \bar{w}+\alpha \hat{w}, \alpha^{2} \bar{p}+\alpha \hat{p}, \alpha^{2} \bar{b}+\alpha \hat{b}\right)$,

where the bar indicates mean and the circumflex indicates the fluctuating part, composed of all wave components. The $\xi$ average will also be indicated with $<\cdot>$. Only the horizontal mean flow $\bar{u}$ is needed for the final amplitude equations. The mean quantities were shown in Grimshaw and McHugh (2013) to be $O\left(\alpha^{2}\right)$, as indicated above.

A discussion of the general equations governing the waveinduced mean flow is given by Andrews and McIntyre (1978a, b) and Grimshaw (1979). In some cases an explicit expression for the mean may be found, as in Dosser and Sutherland (2011). Following Sutherland (2006), Grimshaw and McHugh (2013) found that, for the present configuration and accurate to second order in $\alpha$,

$\bar{u}=\frac{N^{2}}{c_{\mathrm{p}}}<\chi^{2}>$,

$\bar{w}=0$,

where $\chi$ is the vertical displacement. While the averaging in Grimshaw and McHugh (2013) is an $x$ average and therefore somewhat different, it is readily shown that the above expression is valid here with the $\xi$ average. The equation governing the evolution of $\chi$ is

$\frac{\mathrm{D} \chi}{\mathrm{D} t}=w$.

However, the linear version $-c_{\mathrm{p}} \chi_{\xi} \approx w$ is adequate and allows evaluation of $\bar{u}$ using Eqs. (19) and (20). Finally, the mean flow in the lower layer is

$\bar{u}=\bar{u}_{\mathrm{m}}+\left(\bar{u}_{\mathrm{i}} e^{i 2 n_{1} z}+\bar{u}_{\mathrm{i}}^{*} e^{-i 2 n_{1} z}\right), \quad z<0$,

where

$\bar{u}_{\mathrm{m}}=\frac{2}{c_{\mathrm{p}}} \frac{N_{1}^{2}}{\sigma^{2}}\left(I I^{*}+R R^{*}\right)$,

$\bar{u}_{\mathrm{i}}=\frac{2}{c_{\mathrm{p}}} \frac{N_{1}^{2}}{\sigma^{2}} I^{*} R$.

There are two parts to this mean flow. One part is $\bar{u}_{\mathrm{m}}$, which is identical to the mean flow that would be obtained if the incident and reflected wave packets are acting individually, and the mean flows are merely added. The second part is the term containing $\bar{u}_{\mathrm{i}}$, which is caused by the interference between the incident and reflected waves.

In the upper layer,

$\bar{u}=\frac{2}{c_{\mathrm{p}}} \frac{N_{2}^{2}}{\sigma^{2}} T T^{*}, \quad z>0$.

\section{The interaction equations}

The leading order contributions to the primary harmonic in Eqs. (1)-(4) will arise from linear terms and from interactions between the wave perturbations and the mean flow, as in Tabaei and Akylas (2007). Hence the leading-order primaryharmonic terms in Eqs. (1)-(4) are

$$
-c_{\mathrm{p}} \hat{u}_{\xi}+\epsilon \hat{u}_{t_{1}}+\epsilon^{2} \hat{u}_{t_{2}}+\alpha^{2} \bar{u}_{\xi}+\alpha^{2} \bar{u}_{z} \hat{w}=-\frac{1}{\rho_{0}} \hat{p}_{\xi},
$$




$$
\begin{gathered}
-c_{\mathrm{p}} \hat{w}_{\xi}+\epsilon \hat{w}_{t_{1}}+\epsilon^{2} \hat{w}_{t_{2}}+\alpha^{2} \bar{u} \hat{w}_{\xi} \\
=-\frac{1}{\rho_{0}}\left(\hat{p}_{z}+\epsilon \hat{p}_{z_{1}}+\epsilon^{2} \hat{p}_{z_{2}}\right)-\hat{b}, \\
\hat{u}_{\xi}+\hat{w}_{z}+\epsilon \hat{w}_{z_{1}} \epsilon^{2} \hat{w}_{z_{2}}=0 \\
-c_{\mathrm{p}} \hat{b}_{\xi}+\epsilon \hat{b}_{t_{1}}+\epsilon^{2} \hat{b}_{t_{2}}+\alpha^{2} \bar{u} \hat{b}_{\xi}-N^{2} \hat{w}=0 .
\end{gathered}
$$

Note that the quadratic combinations of wave quantities are exactly zero at this order and hence do not appear. Formally eliminating $\hat{u}, \hat{b}$, and $\hat{p}$ from among these equations while dropping terms higher than quadratic in the small parameters results in

$$
\begin{aligned}
& \left(-c_{\mathrm{p}} \frac{\partial}{\partial \xi}+\epsilon \frac{\partial}{\partial t_{1}}+\epsilon^{2} \frac{\partial}{\partial t_{2}}\right)^{2} \\
& {\left[\frac{\partial^{2}}{\partial \xi^{2}}+\left(\frac{\partial}{\partial z}+\epsilon \frac{\partial}{\partial z_{1}}+\epsilon^{2} \frac{\partial}{\partial z_{2}}\right)^{2}\right] \hat{w}} \\
& -\alpha^{2} c_{\mathrm{p}} \frac{\partial^{2}}{\partial \xi^{2}}\left[\bar{u}\left(\hat{w}_{\xi \xi}+\hat{w}_{z z}+\frac{N^{2}}{c_{\mathrm{p}}^{2}} \hat{w}\right)-\bar{u}_{z z} \hat{w}\right]=0 .
\end{aligned}
$$

Using Eqs. (19) and (20) in Eq. (35) and expanding shows that the linear terms combine to zero, as expected. Since the nonlinear terms only appear at second order, then the firstorder terms result in

$$
\left(\frac{\partial}{\partial t_{1}}-c_{\mathrm{g}} \frac{\partial}{\partial z_{1}}\right) I=0
$$

and the same expression acting on $R$ and $T$. The secondorder expression is

$$
\begin{aligned}
& I_{t_{2}}+c_{\mathrm{g}} I_{z_{2}}-i \frac{1}{2} c_{\mathrm{g}}^{\prime} I_{z_{1} z_{1}} \\
& \quad-\frac{\alpha^{2}}{\epsilon^{2}} 2 \sigma\left[\left(k^{2}+n_{1}^{2}\right) k \bar{u}_{\mathrm{m}} I+\left(k^{2}-n_{1}^{2}\right) k \bar{u}_{\mathrm{i}}^{*} R\right]=0, \\
& \quad z<0,
\end{aligned}
$$

where $c_{\mathrm{g}}$ is the vertical group velocity,

$c_{\mathrm{g}}=\sigma \frac{n_{1}}{k^{2}+n_{1}^{2}}, \quad z<0$,

and $c_{\mathrm{g}}^{\prime}$ is a derivative of the group velocity:

$c_{\mathrm{g}}^{\prime}=\frac{\partial c_{\mathrm{g}}}{\partial n_{1}}=\sigma \frac{k^{2}-2 n_{1}^{2}}{\left(k^{2}+n_{1}^{2}\right)^{2}}, \quad z<0$.

Now use the expressions for the mean flow, Eqs. (27) and (30), and revert to original variables $t$ and $z$ to obtain the final amplitude equation for the incident waves:

$$
\begin{aligned}
I_{t} & +c_{\mathrm{g}} I_{z}-i \frac{1}{2} c_{\mathrm{g}}^{\prime} I_{z z} \\
& +i \alpha^{2} \frac{2}{\sigma}\left[\left(k^{2}+n_{1}^{2}\right)\left(|I|^{2}+|R|^{2}\right)+\left(k^{2}-n_{1}^{2}\right)|R|^{2}\right] I \\
& =0, z<0 .
\end{aligned}
$$

Note that $I=O(1)$, since $\alpha$ was inserted into the above expressions. Alternatively, the boundary conditions that create the wave packet could have $I=O(\alpha)$, and then $\alpha$ would not be explicitly present in Eq. (40).

A similar development for the reflected and transmitted waves leads to

$$
\begin{aligned}
& R_{t}-c_{\mathrm{g}} R_{z}-i \frac{1}{2} c_{\mathrm{g}}^{\prime} R_{z z} \\
& \quad+i \alpha^{2} \frac{2}{\sigma}\left[\left(k^{2}+n_{1}^{2}\right)\left(|I|^{2}+|R|^{2}\right)+\left(k^{2}-n_{1}^{2}\right)|I|^{2}\right] \\
& \quad R=0, z<0, \\
& T_{t}+c_{\mathrm{g}} T_{z}-i \frac{1}{2} c_{\mathrm{g}}^{\prime} T_{z z}+i \alpha^{2} \frac{2}{\sigma}\left(k^{2}+n_{2}^{2}\right)|T|^{2} T=0, \\
& \quad z>0,
\end{aligned}
$$

where

$c_{\mathrm{g}}=\sigma \frac{n_{2}}{k^{2}+n_{2}^{2}}, \quad z>0$,

and

$c_{\mathrm{g}}^{\prime}=\frac{\partial c_{\mathrm{g}}}{\partial n_{2}}=\sigma \frac{k^{2}-2 n_{2}^{2}}{\left(k^{2}+n_{2}^{2}\right)^{2}}, \quad z>0$.

The interfacial conditions must be treated in the same manner. The nonlinear terms in both the kinematic Eq. (9) and dynamic Eq. (14) conditions have quadratic and cubic terms that will contribute at second order. The cubic terms have a contribution due to the fact that the second-harmonic velocities at the interface are not zero (unlike the constant $N$ case mentioned above). These second harmonics are treated in McHugh (2009). To account for these, separate the velocity components into a mean velocity, primary harmonic, and secondary harmonic. Hence instead of Eq. (26),

$u=\alpha^{2} \bar{u}+\alpha \hat{u}^{(1)}+\alpha^{2} \hat{u}^{(2)}+\cdots$,

and similar expressions for the other variables.

Keeping only cubic terms, the kinematic interfacial condition is

$\eta_{t}+u \eta_{x}+u_{z} \eta \eta_{x} \approx w+w_{z} \eta+\frac{1}{2} w_{z z} \eta^{2}$,

on $z=0$, where velocities from either layer are used. Now insert Eq. (45) and use Eq. (15), keep only contributions to 
the primary harmonic, and drop higher-order terms as they appear to achieve

$$
\begin{aligned}
& -c_{\mathrm{p}} \hat{\eta}_{\xi}^{(1)}+\epsilon \hat{\eta}_{t_{1}}^{(1)}+\epsilon^{2} \hat{\eta}_{t_{2}}^{(1)} \\
& +\alpha^{2}\left(\bar{u} \hat{\eta}_{\xi}^{(1)}+\hat{u}^{(1)} \hat{\eta}_{\xi}^{(2)}+\hat{u}^{(2)} \hat{\eta}_{\xi}^{(1)}+\hat{u}_{z}^{(1)} \hat{\eta}^{(1)} \hat{\eta}_{\xi}^{(1)}\right) \\
& \approx w^{(1)}+\alpha^{2}\left(\hat{\eta}^{(1)} \hat{w}_{z}^{(2)}+\hat{\eta}^{(2)} \hat{w}_{z}^{(1)}+\frac{1}{2} \hat{w}_{z}^{(1)} \hat{\eta}^{(1) 2}\right) .
\end{aligned}
$$

The dynamic condition is

$$
\left[u_{t}+(u u)_{x}+(u w)_{z}+\eta_{x} w_{t}+\eta u_{z t}+\eta_{x} b-N^{2} \eta \eta_{x}\right]_{-}^{+} \approx 0
$$

on $z=0$. As above, use Eqs. (45) and (15), keep only contributions to the primary harmonic, and drop higher-order terms as they appear to achieve

$$
\begin{aligned}
& {\left[-c_{\mathrm{p}} \hat{u}_{\xi}^{(1)}+\epsilon \hat{u}_{t_{1}}^{(1)}+\epsilon^{2} \hat{u}_{t_{2}}^{(1)}\right.} \\
& +\alpha^{2}\left(-c_{\mathrm{p}}\left[\hat{\eta}^{(1)} \hat{u}_{\xi z}^{(2)}+\hat{\eta}^{(2)} \hat{u}_{\xi z}^{(1)}+\hat{\eta}_{\xi}^{(1)} \hat{w}_{\xi}^{(2)}+\hat{\eta}_{\xi}^{(2)} \hat{w}_{\xi}^{(1)}\right]\right. \\
& +\left[2 \hat{u}^{(1)} \hat{u}^{(2)}\right]_{\xi}+\left[\hat{u}^{(1)} \hat{w}^{(2)}+\hat{u}^{(2)} \hat{w}^{(1)}\right]_{z}+\hat{\eta}_{\xi}^{(1)} \hat{b}^{(2)}+\hat{\eta}_{\xi}^{(2)} \hat{b}^{(1)} \\
& -\frac{1}{2} c_{\mathrm{p}} \hat{\eta}^{(1) 2} \hat{u}_{\xi}^{(1)}+\hat{\eta}^{(1)}\left[\hat{u}^{(1) 2}\right]_{\xi z}+\hat{\eta}^{(1)}\left[\hat{u}^{(1)} \hat{w}^{(1)}\right]_{z z} \\
& +\hat{\eta}^{(1)} \hat{\eta}_{\xi}^{(1)}\left[-c_{\mathrm{p}} \hat{w}_{\xi z}^{(1)}+\hat{b}_{z}^{(1)}\right]+\hat{\eta}_{\xi}^{(1)}\left[\hat{u}^{(1)} \hat{w}^{(1)}\right]_{\xi}+\hat{\eta}_{\xi}^{(1)}\left[\hat{w}^{(1) 2}\right]_{z} \\
& \left.\left.-N^{2}\left[\hat{\eta}^{(1)} \hat{\eta}_{\xi}^{(2)}+\hat{\eta}^{(2)} \hat{\eta}_{\xi}^{(1)}\right]\right)\right]_{-}^{+} \approx 0
\end{aligned}
$$

on $z=0$. Insert Eqs. (19) and (20) along with associated expressions for the horizontal component of velocity into Eqs. (47) and (49) and simplify to achieve a relationship between the reflected and transmitted wave amplitudes $-R$ and $T$ - and the incident wave amplitude $I$ at the interface:

$$
\begin{aligned}
& R+\epsilon \frac{i}{\sigma} R_{t_{1}}=\left[\frac{n_{1}-n_{2}}{n_{1}+n_{2}}\right]\left[I+\epsilon \frac{i}{\sigma} I_{t_{1}}\right]+O\left(\alpha^{2}\right), \\
& T+\epsilon \frac{i}{\sigma} T_{t_{1}}=\left[\frac{2 n_{1}}{n_{1}+n_{2}}\right]\left[I+\epsilon \frac{i}{\sigma} I_{t_{1}}\right]+O\left(\alpha^{2}\right) .
\end{aligned}
$$

The Schrodinger equation for the incident waves written in terms of slow variables, Eq. (37), has all terms of the same order (since $\alpha^{2} / \epsilon^{2}=O(1)$ ). Hence dispersion and nonlinearity are in balance, and all higher-order terms are neglected. It would seem that the matching form of the interfacial conditions written in terms of slow variables should also retain only terms that are in balance, and any terms containing a small parameter should be neglected. The result is that Eqs. (50) and (51) are replaced with their linear forms, Eqs. (22) and (23):

$$
\begin{aligned}
& R=\mathcal{R} I, \\
& T=\mathcal{T} I,
\end{aligned}
$$

on $z=0$.
However, the results given below show that the vertical integral of $\bar{u}$ is only approximately conserved when the wave packet transits the interface, indicating that momentum is only approximately conserved during this time. This integral is very accurately conserved before the packet reaches the interface; hence the error can only be reduced with alternative interfacial conditions. Such an alternative interfacial condition requires the nonlinear terms shown above. Unfortunately these nonlinear interfacial terms have great complexity and hence are not included here. The linear interfacial conditions that are employed here are sufficiently accurate for the present results.

Note that these linear conditions imply continuity of velocity of the wave components. Importantly the total velocity is not necessarily equal at the interface as the mean flow may not be continuous.

\section{Results}

\subsection{Preliminaries}

The amplitude Eqs. (40), (41), and (42) are solved here numerically. Spatial derivatives are evaluated with second-order central differences. Temporal integration is achieved with the fourth-order Adams-Bashforth method, resulting in explicit algebraic equations (see Ames (1977) for a general discussion). The boundary point at the end of the domain is treated with the second-order upwind scheme. This onesided method allows waves to exit the region without reflections (the reflected waves have already been treated in the derivation of the amplitude equations). Single-layer cases discussed below employ a total resolution of 1600 grid points while two-layer cases use 800 grid points in each layer, which was found to be adequate for all parameter values. The depth of each layer for two-layer cases was set to $4 / 3$ of the packet length, which also sets the grid spacing. Several cases were treated with double this resolution to confirm convergence. The time step was set to avoid numerical instability, generally being in the range $0.0001<\Delta t<0.001$.

All variables are rescaled with the horizontal wavenumber $k$ and the buoyancy frequency in the lower layer $N_{1}$. The buoyancy frequency ratio for two-layer cases is chosen to have the value $N_{2} / N_{1}=2$, matching approximately Earth's tropopause. The results then depend on three parameters: $\epsilon$, $\alpha$, and $n_{1} / k$.

A wave envelope is created at the bottom boundary by imposing the value of the real part of $I$ to be the raised cosine function:

$$
\frac{k I}{N_{1}}=\frac{1}{2}\left[1-\cos \left(2 \pi \epsilon c_{\mathrm{g}} t\right)\right],
$$

$t \in\left[0,1 / \epsilon c_{\mathrm{g}}\right]$. Other wave packet shapes, such as a Gaussian shape, have been considered and produce the same general results. 


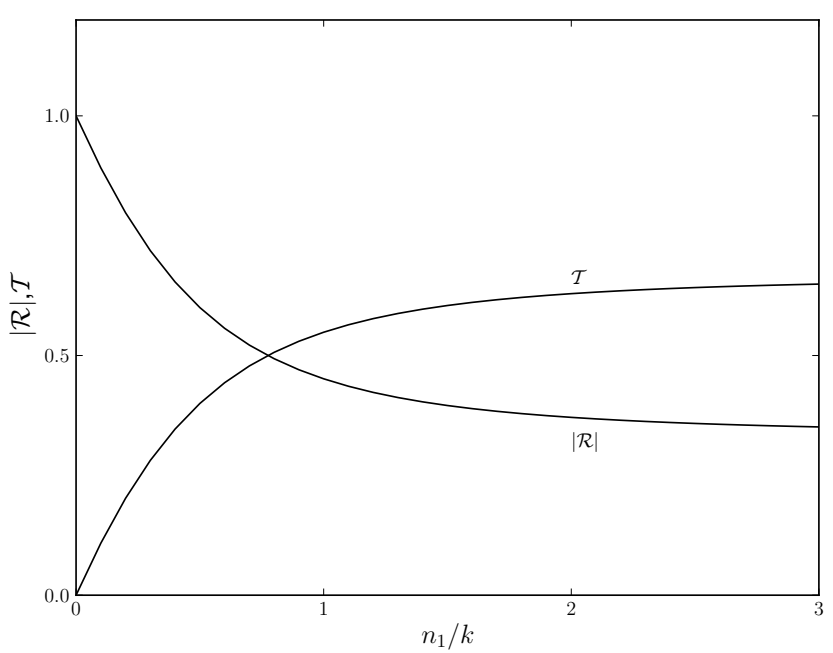

Figure 1. Transmission coefficient $\mathcal{T}$ and absolute value of the reflection coefficient $|\mathcal{R}|$ as $n_{1} / k$ increases.

The value of $\alpha$ is set to $\alpha=0.1$. Larger values of $\alpha$ mean stronger nonlinear effects, with consequences that depend on the value of $n_{1} / k$. The value of $\epsilon$ is set to $\epsilon=0.025$. The parameter $\epsilon$ measures how slowly the wave parameters are changing, and its value controls the dispersion. The definition of $\epsilon$ is the inverse of the packet length, and the above raised cosine shape has the obvious choice of overall length. Sutherland (2006) and Tabaei and Akylas (2007) used a Gaussian packet shape, $e^{-z^{2} / 2 \sigma^{2}}$, and defined the packet length to be $1 / \sigma$. Comparing these two packet shapes, if they have the same full width at half maximum (FWHM), then the overall length of the raised cosine is $4 \sqrt{2 \ln 2}$ times the value of $1 / \sigma$ for the Gaussian profile. This means that $\epsilon=0.025$ used here is approximately the same as the value $\epsilon=0.1$ in Sutherland (2006) and Tabaei and Akylas (2007). The derivation of the nonlinear Schrodinger equations implies a balance between nonlinearity and dispersion, suggesting that $\alpha \approx \epsilon$. However the simulations show that with $\epsilon=0.1$ and a raised cosine shape, dispersion dominates and nonlinearity is unimportant. Thus the choice of the value for $\epsilon$ is made here to achieve an approximate balance in the results.

The behavior of the waves with increasing $n_{1} / k$ is complicated and depends partly on the value of the reflection and transmission coefficients $\mathcal{R}$ and $\mathcal{T}$ given by Eqs. (24) and (25) (see Fig. 1). For very small values of $n_{1} / k, \mathcal{R}$ approaches unity while $\mathcal{T}$ approaches zero, indicating that the waves are nearly perfectly reflected. As $n_{1} / k$ increases, $\mathcal{R}$ approaches the value $1 / 3$ while $\mathcal{T}$ approaches $2 / 3$. Hence for large values of $n_{1} / k$, the reflection and transmission coefficients are approximately constant. Perfect transmission never occurs for any value of $n_{1} / k$.

The behavior with increasing $n_{1} / k$ also depends greatly on the strength of the mean flow. The results show that the mean flow given in Eqs. (27) and (30) increases strongly with

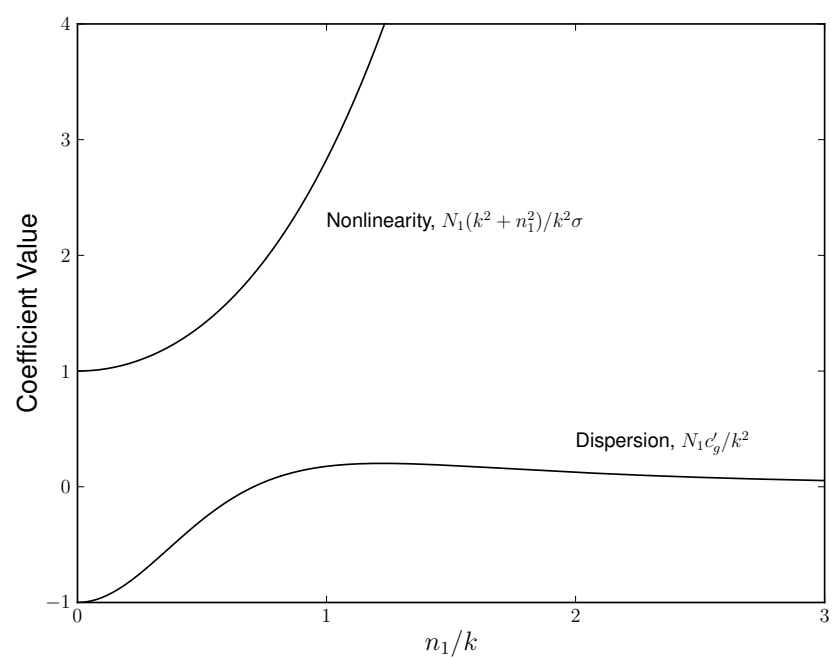

Figure 2. Behavior of the coefficients of the dispersion and nonlinear terms in the amplitude equations as $n_{1} / k$ increases.

$n_{1} / k$, as measured approximately by the factor $\frac{2 N_{1}^{2}}{c_{\mathrm{p}} \sigma^{2}}$. This increase is primarily due to the direction of the group velocity becoming more horizontal as $n_{1} / k$ increases. Rescale this coefficient with $k / N_{1}$, resulting in a quantity that is defined as $U$ :

$U=\frac{k}{N_{1}}\left(\frac{2 N_{1}^{2}}{c_{\mathrm{p}} \sigma^{2}}\right)=2\left(\frac{k^{2}+n_{1}^{2}}{k^{2}}\right)^{3 / 2}$.

Profiles of the mean flow velocity appearing in the figures are normalized by $U$.

The coefficient of the nonlinear term and the dispersion term in the amplitude equation governing the incident waves Eq. (40) are plotted in Fig. 2. The coefficient value for the nonlinear term can be seen to increase strongly with $n_{1} / k$, as a direct result of the mean flow dependence on $n_{1} / k$. Hence stronger nonlinear effects are expected as $n_{1} / k$ increases.

The dispersion coefficient is negative for small values of $n_{1} / k$ and changes sign at $n_{1} / k=1 / \sqrt{2}$. As discussed by Sutherland (2001), the waves experience a modulational instability in the region where this coefficient is negative. As a result of this instability, a wave packet will initially become more focused and grow in amplitude. This dispersion coefficient is positive for $n_{1} / k>1 / \sqrt{2}$ and reaches a maximum at $n_{1} / k=\sqrt{2}$. When the coefficient is positive, the wave packet defocuses.

\subsection{Properties of the amplitude equations}

Some insight is revealed by separating the wave amplitude into a magnitude and phase,

$I=|I| e^{i \phi}$, 
where $\phi$ is the phase of the incident waves. Using this in Eq. (40) and separating real and imaginary parts results in

$$
\begin{aligned}
& |I|_{t}+c_{\mathrm{g}}|I|_{z}+\frac{1}{2} c_{\mathrm{g}}^{\prime}\left[|I| \phi_{z z}+2|I|_{z} \phi_{z}\right]=0, \\
& \phi_{t}+c_{\mathrm{g}} \phi_{z}+\frac{1}{2} c_{\mathrm{g}}^{\prime}\left[\frac{|I|_{z z}}{|I|}-\phi_{z}^{2}\right] \\
& \quad+\alpha^{2} \frac{1}{2}\left[\left(k^{2}+n_{1}^{2}\right)\left(|I|^{2}+|R|^{2}\right)+\left(k^{2}-n_{1}^{2}\right)|I|^{2}\right]=0 .
\end{aligned}
$$

Similar equations can be obtained for the other wave packets. Equation (56) governs the evolution of the magnitude of the incident wave $|I|$. The influence of the reflected wave $R$ does not directly appear in Eq. (56). Hence when incident and reflected waves overlap, the reflected wave only affects the incident wave packet shape as a result of the evolution of $\phi$ in the dispersion term. For cases where the dispersive effect is zero or negligible, then Eq. (56) becomes

$$
|I|_{t}+c_{\mathrm{g}}|I|_{z}=0
$$

indicating that the wave packet propagates with the group velocity $c_{\mathrm{g}}$ but without any evolution in the packet shape, and unaffected by the reflected wave even when overlapping.

Multiply Eq. (56) by $|I|$ and rearrange to obtain

$$
\left(\frac{\partial}{\partial t}+c_{\mathrm{g}} \frac{\partial}{\partial z}\right)|I|^{2}+c_{\mathrm{g}}^{\prime} \frac{\partial}{\partial z}\left(|I|^{2} \phi_{z}\right)=0 \text {. }
$$

For time periods before the incident wave packet reaches the interface, this may be integrated to produce

$$
\frac{\partial}{\partial t} \int|I|^{2} \mathrm{~d} z=0
$$

where boundary terms are zero since $|I|$ is zero outside the wave packet. Equation (59) shows that the spatial integral of $|I|^{2}$ is conserved in a layer of constant $N$, despite the influence of dispersion and nonlinearity. Once the incident wave packet reaches the interface, the incident wave packet begins to decrease in area as the reflected and transmitted waves are created. The combination of Eq. (56), which shows that $|I|$ is not influenced by nonlinear effects, and Eq. (59), which shows that the area of the wave packet is limited, suggest that the incident wave packet shape will decrease monotonically during this time.

Equation (57) governs the incident wave phase, and does explicitly contain $R$. The effect of the dispersion term is complicated and will be determined with numerical results. However the nonlinear term in Eq. (57) is positive, and in the absence of dispersion,

$$
\left(\frac{\partial}{\partial t}+c_{\mathrm{g}} \frac{\partial}{\partial z}\right) \phi<0
$$

Hence the nonlinear effect causes the phase to decrease monotonically.
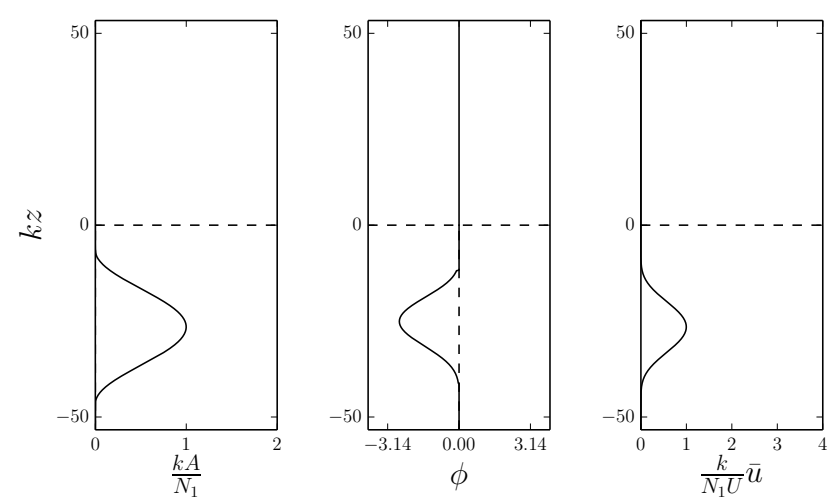

(a) $N_{1} \tau \approx-75$
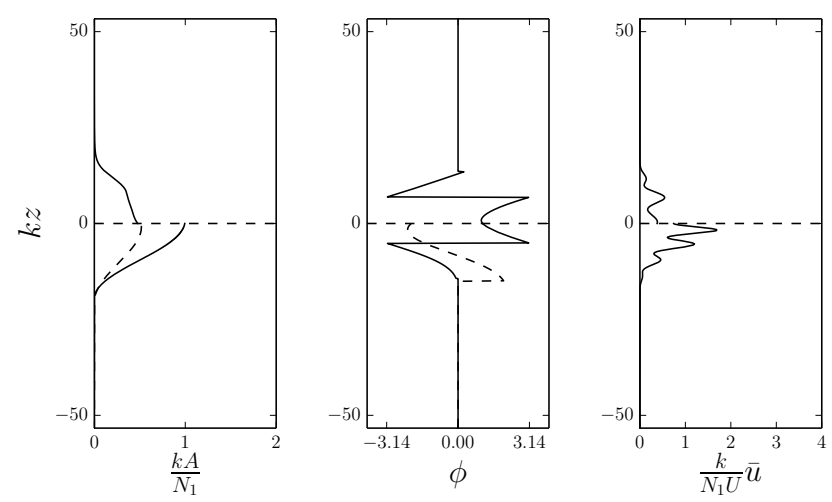

(b) $N_{1} \tau \approx 0$
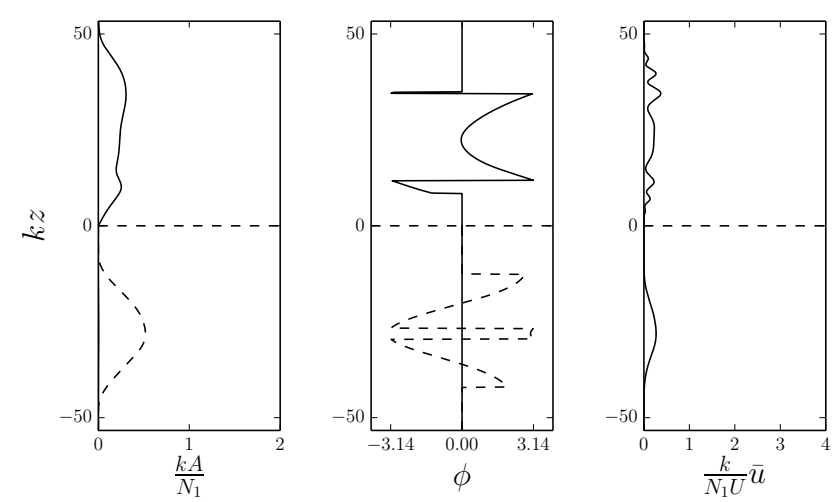

(c) $N_{1} \tau \approx+75$

Figure 3. Vertical profiles of the wave magnitudes $A$ (left panel), phases $\phi$ (center panel), and mean flow (right panel) at three times in two layers. The parameter values are $n_{1} / k=1 / \sqrt{2}, N_{2} / N_{1}=2$, $\epsilon=0.025$, and $\alpha=0.1$. The dashed profiles are the magnitude and phase for the downward-moving wave packet (the reflected wave), and the solid profiles are the upward-moving waves (incident and transmitted waves).

\subsection{A dispersion-free case}

For the numerical results, first consider $n_{1} / k=1 / \sqrt{2}$. This special value of $n_{1} / k$ has a zero value for the coefficient of the dispersion term. Sutherland (2006) discussed this case for 
a single layer with constant $N$ and included a third-derivative dispersive term that appears at the next order, suggesting that wave dispersion is still important for this parameter value; however it is not captured with the above amplitude equations. While the results shown below for $n_{1} / k=1 / \sqrt{2}$ are lacking dispersion, this parameter value is still included here to emphasize that the interfacial dynamics occur even without dispersion.

Figure 3 shows vertical profiles of wave amplitude and mean flow at three times for $n_{1} / k=1 / \sqrt{2}$. Note that a time value of zero here corresponds to the incident wave packet centered at the origin, which is also the mean position of the interface, as indicated with a dashed line. The BruntVäisälä frequency ratio is $N_{2} / N_{1}=2$. Each time value in Fig. 3 shows three panels, which contain (from left to right in each subfigure) a vertical profile of the wave magnitude, the wave phase, and the wave-induced mean flow. The wave packet moves vertically without any significant change in shape before reaching the interface, as predicted by Eq. (58). The dashed profiles are the magnitude and phase for the downward-moving wave packet (the reflected wave), and the solid profiles are the upward-moving waves (incident and transmitted waves). Note that there is only a single profile for the mean flow in the lower layer (e.g., no dashed profile) as it is due to the combination of incident and reflected waves.

Figure $3 \mathrm{~b}$ has all three wave packets present simultaneously. The mean flow at this stage shows the striking discontinuity at the interface. The reason for this discontinuity is that in the lower layer the mean flow is driven by both incident and reflected wave. Since they both have a horizontal component of group velocity that is positive, then the mean flow they generate is positive, despite having a vertical group velocity that has opposite sign. In contrast, the upper layer only has the transmitted wave driving a mean flow and is therefore always weaker at the interface.

The mean flow in Fig. $3 \mathrm{~b}$ also shows oscillations that are not present before the packet reaches the interface. These oscillations are due to the interference mean given by Eqs. (27) and (29). The interference mean only occurs under the mean position of the interface, and only when the incident wave and reflected wave are overlapping. Thus the oscillations in $\bar{u}$ above the interface are due to the nonlinear coupling of the three wave packets.

\subsection{Dispersive evolution}

The coefficient of the dispersion term is negative when $n_{1} / k<1 / \sqrt{2}$. The evolution of the wave packet for such a case is shown in Fig. 4 for a single layer with constant $N$ and $n_{1} / k=0.4$. This same single-layer case was previously treated by Sutherland $(2001,2006)$ and Tabaei and Akylas (2007). Figure 4a shows that the wave amplitude and the associated mean flow are initially focusing energy toward the center of the wave packet. Figure $4 \mathrm{~b}$ and c show that at later times the peak in wave amplitude has decreased and
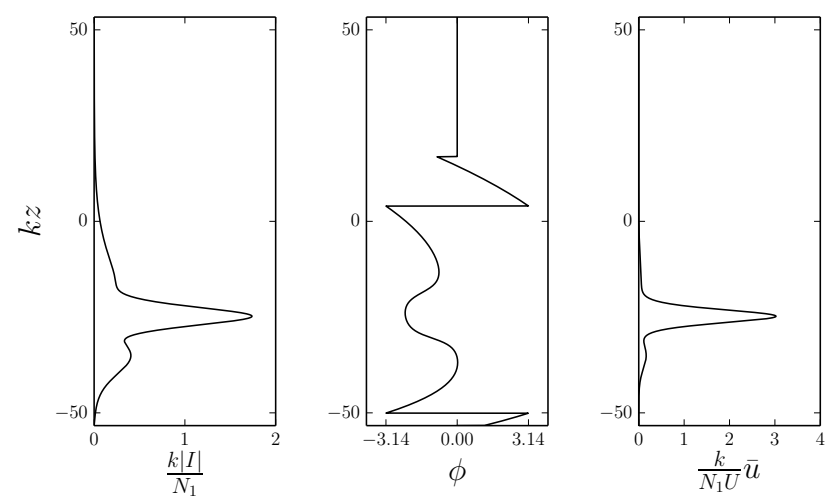

(a) $N_{1} \tau \approx-80$
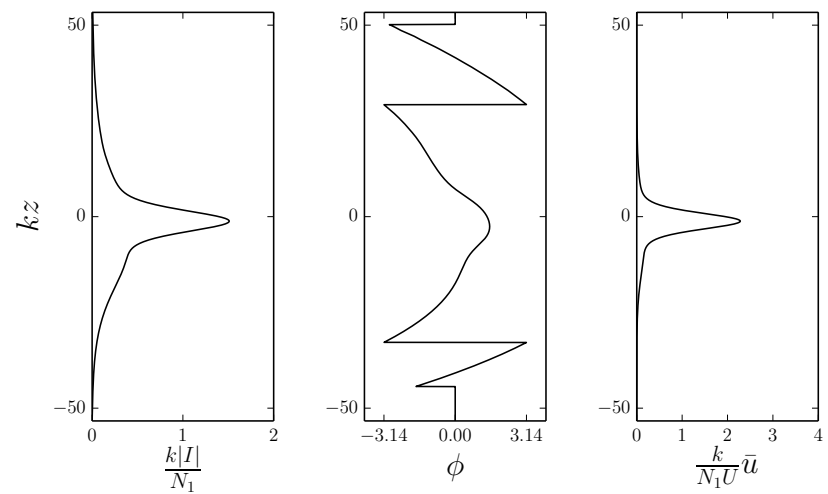

(b) $N_{1} \tau \approx 0$
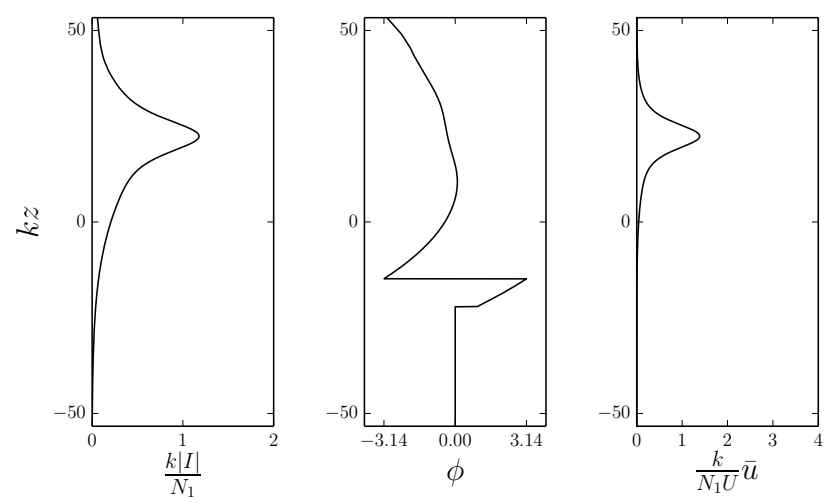

(c) $N_{1} \tau \approx+80$

Figure 4. Vertical profiles of the wave magnitudes $A$ (left panel), phases $\phi$ (center panel), and mean flow (right panel) at three times in a single layer of constant $N$ with $n_{1} / k=0.4, \epsilon=0.025$, and $\alpha=0.1$.

the wave packet has spread significantly. Even longer times would show the focusing effect reappearing. This behavior is due to the modulational instability as discussed by Sutherland (2001). Figure 5a provides filled contours of vertical velocity for the same time value as Fig. 4a, demonstrating the same general pattern as was found by Sutherland (2006). 


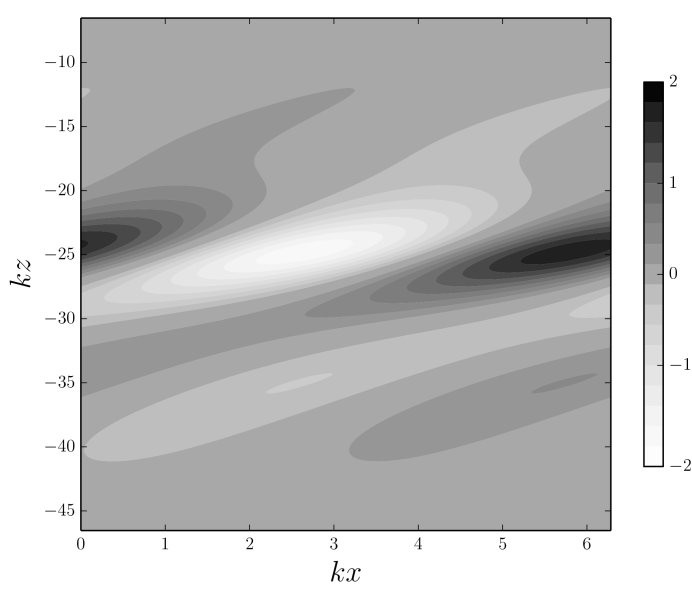

(a) $N_{1} \tau \approx-80$

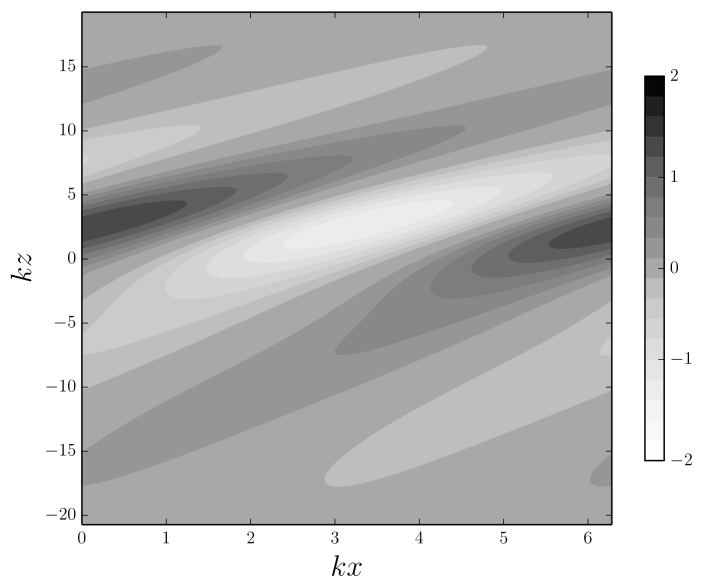

(b) $N_{1} \tau \approx 0$

Figure 5. Contours of vertical velocity in a single layer of constant $N$ with $n_{1} / k=0.4, \epsilon=0.025$, and $\alpha=0.1$.

Figure $5 \mathrm{~b}$ shows the pattern at a later time after the wave amplitude has reached its peak and is decreasing.

The two-layer case with $n_{1} / k=0.4$ is shown in Fig. 6 . Figure $6 \mathrm{a}$ is before the incident wave packet has interacted significantly with the interface, and again the results are the same as the constant $N$ case (Fig. 4a). In Fig. 6b the wave packet is straddling the interface. The mean flow (right panel) again shows the oscillations under the interface that is the interference part of the mean flow $\bar{u}_{\mathrm{i}}$, although these oscillations are somewhat difficult to see due to the different scale on the abscissa. Also evident in Fig. 6b are matching oscillations in $I$ (solid line for both magnitude and phase). These oscillations would not exist without dispersion, as $I$ obeys Eq. (58) without dispersion. Note that these oscillations in $I$ were not present with $n_{1} / k=1 / \sqrt{2}$ in Fig. 3b.

Figure $6 \mathrm{c}$ shows the results at a later time after the reflected and transmitted wave packets are mostly created. The magnitude of $I$ (solid line, left panel) still shows signifi-
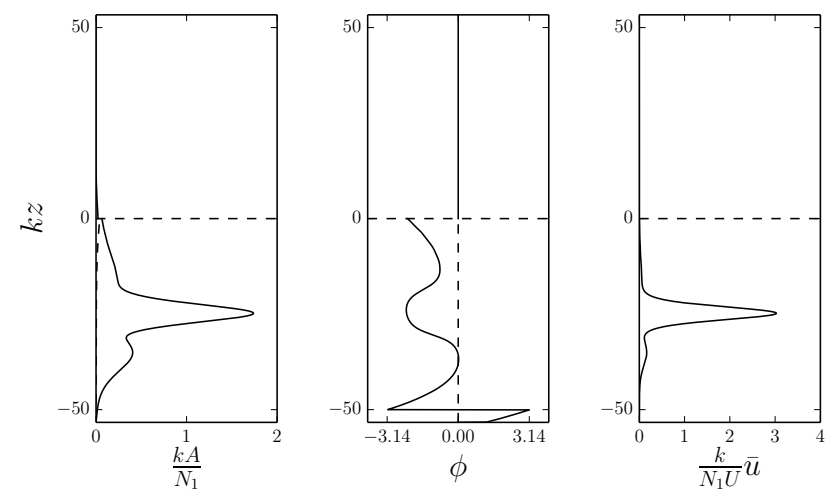

(a) $N_{1} \tau \approx-80$
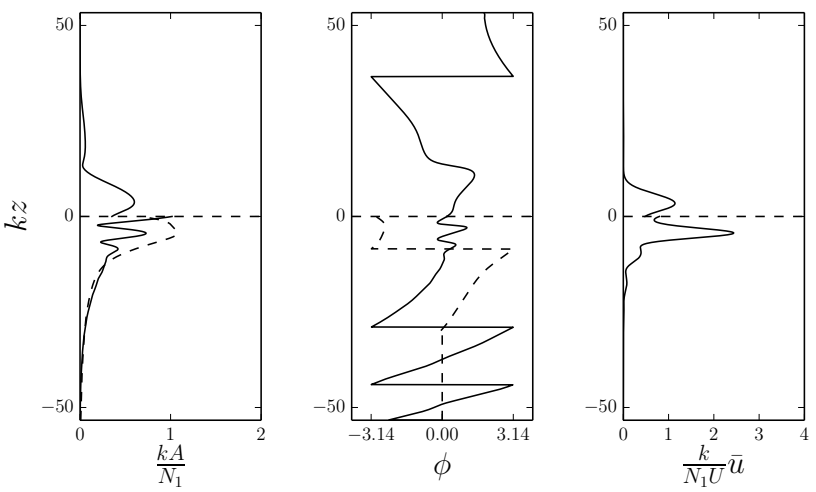

(b) $N_{1} \tau \approx 0$
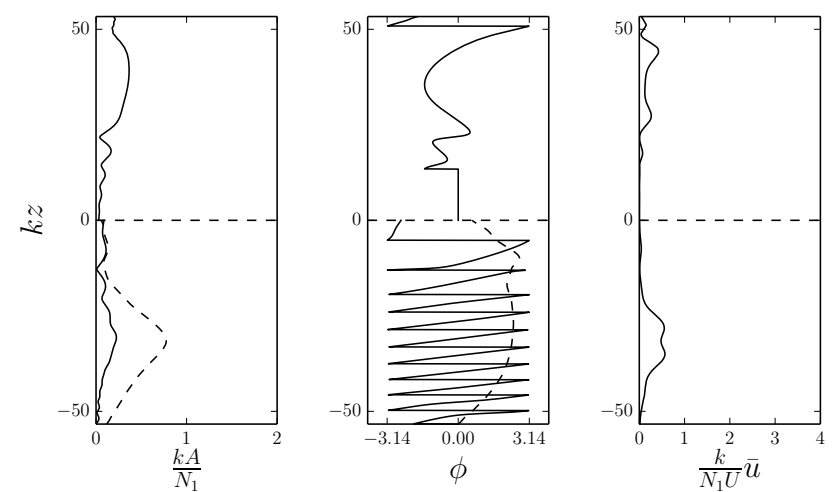

(c) $N_{1} \tau \approx+80$

Figure 6. Vertical profiles of the wave magnitudes $A$ (left panel), phases $\phi$ (center panel), and mean flow (right panel) at three times in two layers. The parameter values are $n_{1} / k=0.4, N_{2} / N_{1}=2$, $\epsilon=0.025$, and $\alpha=0.1$. The line conventions are the same as Fig. 3 .

cant oscillations, while the magnitude of $R$ (dashed line, left panel) does not. The mean flow also has matching oscillations. The incident waves $I$ can only move upwards here; hence these oscillations in $I$ are part of the tail of the incident wave packet. Dispersion has caused the incident wave packet to spread, which continues to interact with the downwardmoving reflected wave packet. For this choice of parame- 


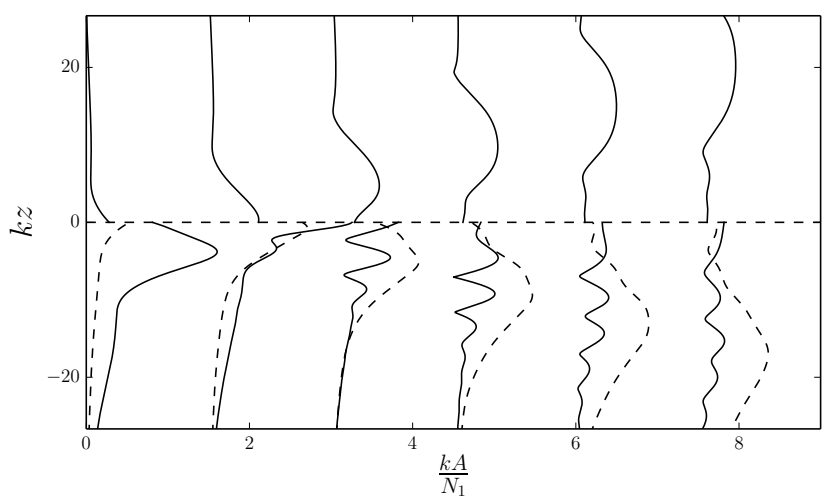

Figure 7. Vertical profiles of the wave magnitude in two layers for a sequence of times. Each profile is shifted by a value of 1.5 for display. The parameter values are $n_{1} / k=0.4, N_{2} / N_{1}=2, \epsilon=0.025$, and $\alpha=0.1$. The line conventions are the same as Fig. 3 .

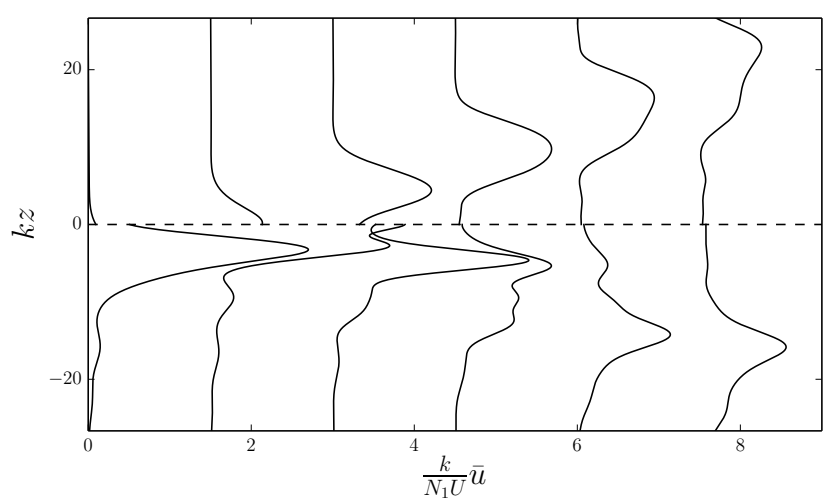

Figure 8. Vertical profiles of the mean flow in two layers for a sequence of times. Each profile is shifted by a value of 1.5 for display. The parameter values are $n_{1} / k=0.4, N_{2} / N_{1}=2, \epsilon=0.025$, and $\alpha=0.1$.

ters $(\epsilon=0.025, \alpha=0.1)$, the incident wave amplitude $I$ had reached its maximum before interacting with the interface and is decreasing while the packet is spreading. This feature of the wave packet dynamics seems to enhance the interaction between the reflected waves and the tail of the incident packet.

Figures 7 and 8 show a close-up view of the wave amplitudes and mean flow near the interface. Figure 7 shows clearly the formation of these smaller-scale oscillations in $I$ and seems to suggest that they are moving downward. However they are not moving downward but are becoming energized by the downward-moving reflected wave $R$.

Figure 9 shows filled contours of vertical velocity, corresponding to the constant $N$ case in Fig. 5b. The wave pattern under the interface in Fig. 9 (the interface is at the ordinate center) with incident and reflected waves overlapping is nearly that of a standing wave.

Figures 10 and 11 show results for a case when the coefficient of dispersion is positive, $n_{1} / k=1$. Figure 10 is the

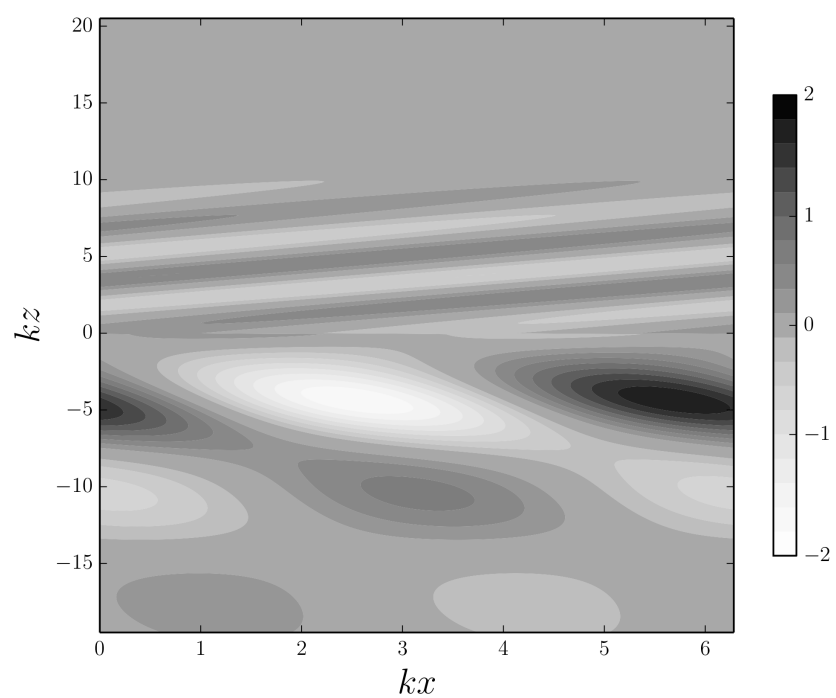

Figure 9. Contours of vertical velocity at $\tau \approx 0$ in two layers. The parameter values are $n_{1} / k=0.4, N_{2} / N_{1}=2, \epsilon=0.025$, and $\alpha=0.1$.

single-layer case with constant $N$ while Fig. 11 is the corresponding two-layer configuration with $N_{2} / N_{1}=2$. Figure 10 shows that as the wave packet ascends in a single layer, the packet shape tends to spread. The two-layer results in Fig. 11b show that the mean flow is again discontinuous as the wave packet interacts with the interface, and that the interference mean is again present. Oscillations in $I$ appear and spread downward, as before. However the dispersion effect is strong here and the transmitted and reflected wave packets are much reduced in strength as a result.

The amplitude and phase obey Eqs. (56) and (57). One of the nonlinear terms in Eq. (57) is directly attributed to the interference mean flow and has the rescaled coefficient

$1-\frac{n_{1}^{2}}{k^{2}}$.

If $n_{1} / k=1$, then this coefficient is zero, and hence this nonlinear term does not contribute to the evolution of $\phi$, making $n_{1} / k=1$ a special case. The influence of the interference part of the mean flow is still felt but indirectly through the other nonlinear terms in Eqs. (56) and (57).

Figures 12 and 13 again show a close-up view of a sequence of profiles of the wave magnitude and corresponding mean flow as the packet transits the interface. Figure 13 shows the development of the discontinuity in the mean flow as well as the appearance of the interference mean flow. Another feature evident in Fig. 13 is that the oscillations in the mean flow under the interface are decreasing in length as time increases. The form of Eq. (27) suggests that the oscillation length should be simply $2 \pi / 2 n_{1}$, which it is when these oscillations first appear. However the nonlinear combination of $I$ and $R$ alters this length. The effect is present for previous cases but is not as prominent. 

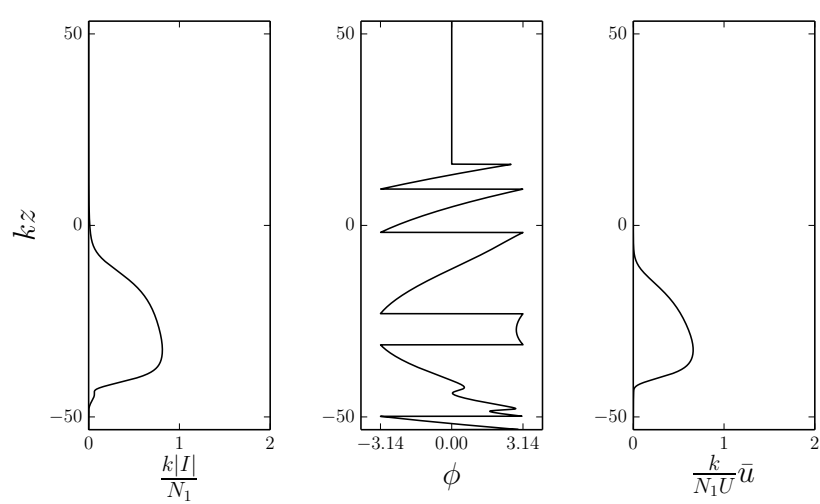

(a) $N_{1} \tau \approx-77$
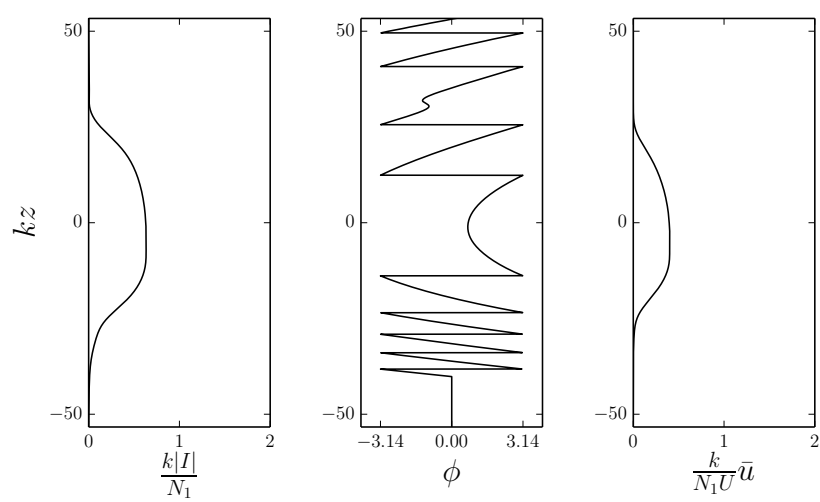

(b) $N_{1} \tau \approx 0$
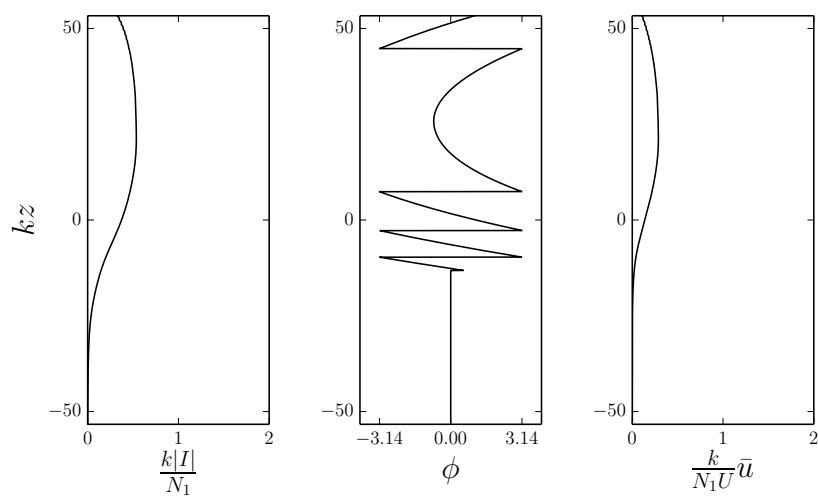

(c) $N_{1} \tau \approx+77$

Figure 10. Vertical profiles of the wave magnitude (left panel), phase (center panel), and mean flow (right panel) at three times in a single layer of constant $N$ with $n_{1} / k=1, \epsilon=0.025$, and $\alpha=0.1$.

Figure 14 shows results for the two-layer case with $n_{1} / k=\sqrt{2}$. This value of $n_{1} / k$ corresponds to the maximum value of the dispersion coefficient in the amplitude equations for the lower layer. With such strong dispersion, the wave packet spreads considerably by the time the packet reaches the interface. The numerical results indicate that with such strong dispersion, increasing the value of $\alpha$ weakens the in- terference mean and the tendency for these oscillations in the mean flow to shorten.

\subsection{Mean flow comparisons}

The overall mean-flow strength is shown in Figs. 15-17 for three examples. Each of these figures show a time evolution of the maximum of the wave packet amplitude. In Fig. 15, $n_{1} / k=1 / \sqrt{2}$ and the dispersion is zero in the lower layer. For this case the maximum value of the wave amplitude is constant until the waves interact with the interface. While the packet is near the interface, the mean flow is enhanced due to the combination of the incident and reflected waves, and also the interference mean. Figure 15 shows that the mean flow is enhanced by a factor of approximately 1.75 at the peak. Figure 16 has $n_{1} / k=0.4$ and shows that the maximum value is nearly four times the value of the mean flow early in the simulation. Also evident here is the oscillation in the tail of the incident wave packet.

Figure 17 has $n_{1} / k=\sqrt{2}$ where the dispersion coefficient is positive and maximum. Here the wave packet is defocusing, resulting in a decrease in the maximum value as the wave packet ascends. The increase in mean at the interface is not strong enough to overcome this decrease, and hence the maximum value of mean flow is at the beginning of the simulation. Overall, Figs. 15-17 indicate that smaller values of $n_{1} / k$ are more likely to have an enhanced jet-like mean flow under the interface.

Also in Figs. $15-17$ is a dashed line showing the velocity difference at the interface and a thick solid line showing the interference mean maximum. In Figs. 15 and 17, these two quantities have approximately the same magnitude. However the velocity difference is not caused by the interference mean; rather they are both caused by the overlapping of the incident and reflected wave packets and hence have approximately the same strength. Note the velocity difference for $n_{1} / k=0.4$ in Fig. 16 is substantially stronger than the interference mean in Fig. 15.

Figure 18 shows a time history of the vertical integral of $\bar{u}$ for two cases: $n_{1} / k=0.4$ and $n_{1} / k=\sqrt{2}$. Once the wave packet is formed, this integral should be constant. Before the packet has interacted with the interface, Fig 18 shows such a constant value for $n_{1} / k=0.4$. With $n_{1} / k=\sqrt{2}$, there is a relatively small decrease in strength of the mean flow, approximately $15 \%$. With $n_{1} / k=0.4$ there is briefly a $25 \%$ error approximately. While this seems large, the maximum value of $\bar{u}$ in Fig. 16 has reached nearly four times the original value, and by comparison this error in the integral of $\bar{u}$ is deemed acceptable.

\subsection{The rigid lid case}

If the interface is replaced with a rigid lid, then the waves are completely reflected but otherwise behave in the same manner as above. The interfacial boundary conditions are re- 

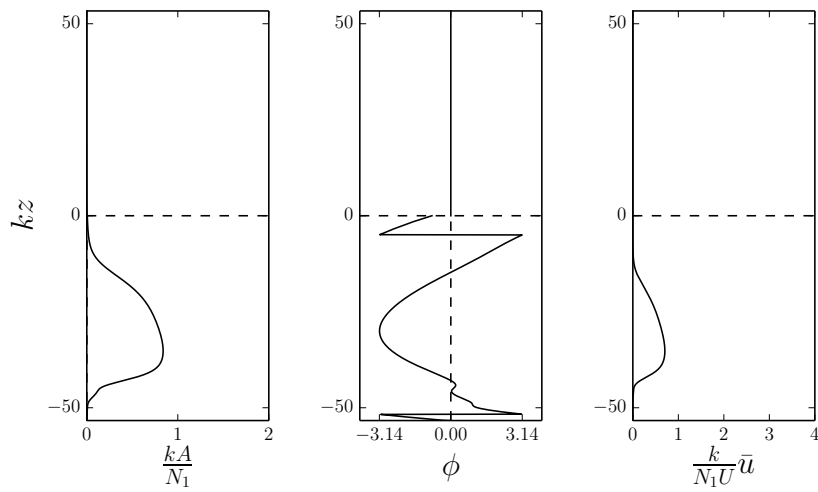

(a) $N_{1} \tau \approx-77$
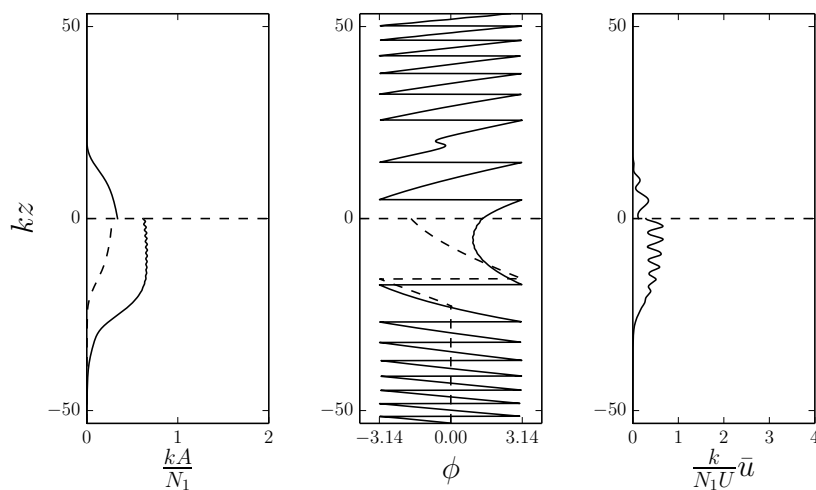

(b) $N_{1} \tau \approx 0$

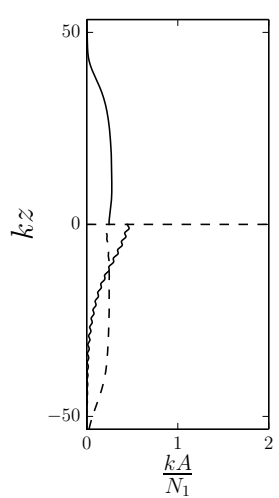

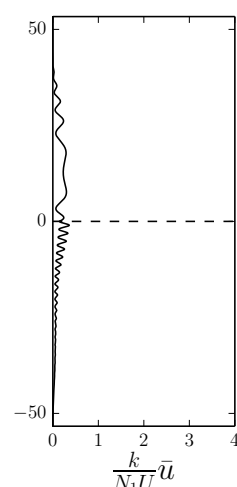

(c) $N_{1} \tau \approx+77$

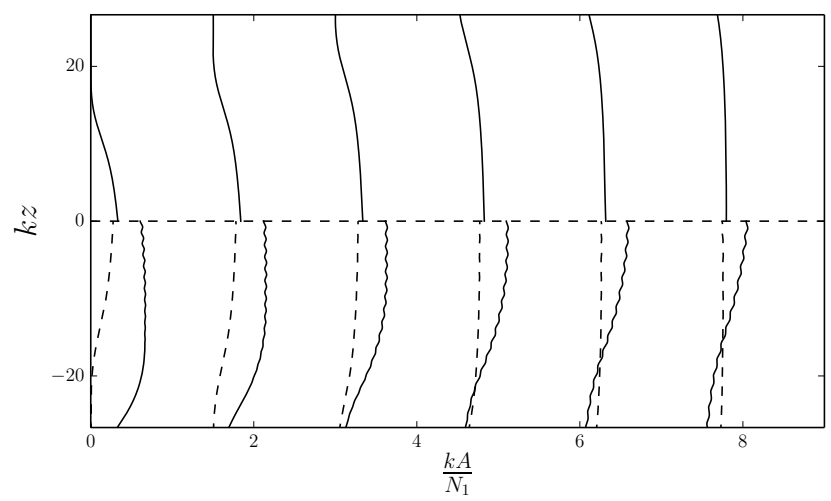

Figure 12. Vertical profiles of the wave magnitude in two layers for a sequence of times. Each profile is shifted by a value of 1.5 for display. The parameter values are $n_{1} / k=1, N_{2} / N_{1}=2, \epsilon=0.025$, and $\alpha=0.1$.

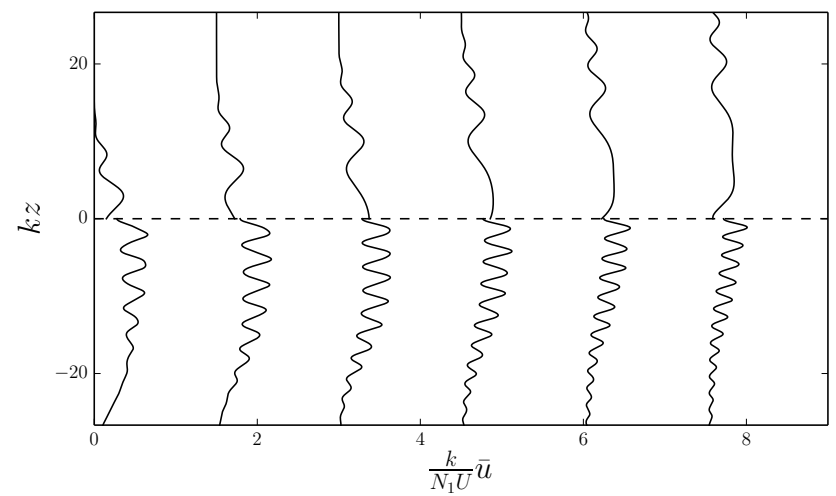

Figure 13. Vertical profiles of the mean flow in two layers for a sequence of times. Each profile is shifted by a value of 1.5 for display. The parameter values are $n_{1} / k=1, N_{2} / N_{1}=2, \epsilon=0.025$, and $\alpha=0.1$. The line conventions are the same as Fig. 3 .

on $z=0$. The incident and reflected wave amplitudes are still governed by Eqs. (40) and (41), while the mean flow is still determined with Eq. (27).

An example case with $n_{1} / k=1 / \sqrt{2}$ is shown in Fig. 19 with vertical profiles of wave magnitude and phase and the mean flow at three time steps, as before. Notice in Fig. 19 that the wave magnitude and mean flow profiles for the upwardmoving packet $\left(N_{1} t \approx-65\right)$ match the corresponding profiles for the downward-moving packet $\left(N_{1} t \approx+65\right)$. The only difference other than the direction of propagation is the phase, which is more oscillatory. The mean flow for $N_{1} \tau \approx 0$ is much stronger than the above case with an interface as a result of the much stronger reflected wave. The maximum of the mean flow is now approximately three times the mean flow of the incident wave packet, compared to a factor of 1.75 in the case with the interface. The interference mean flow is still clearly present with the rigid lid. 

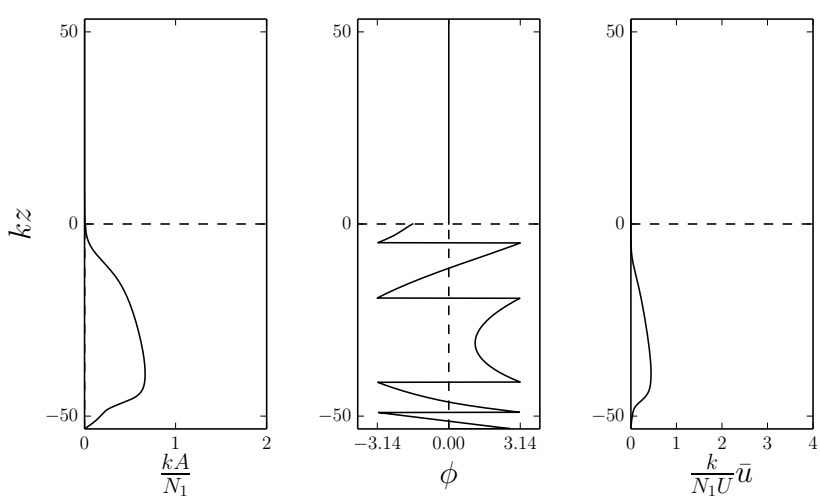

(a) $N_{1} \tau \approx-98$
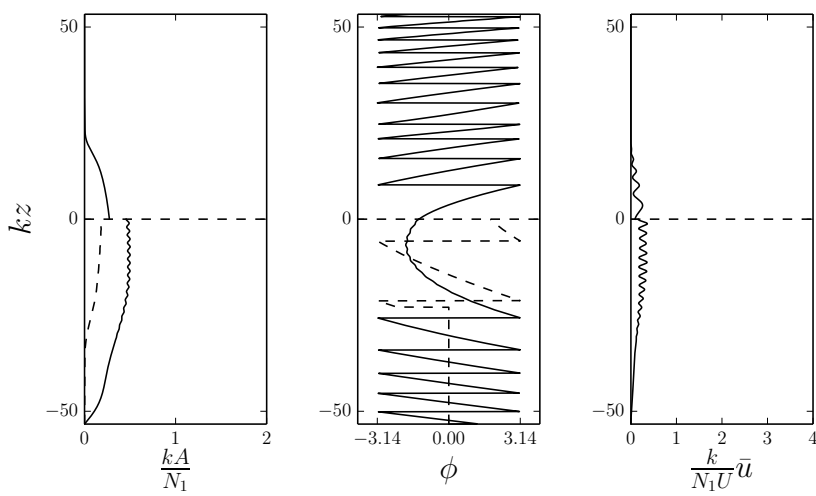

(b) $N_{1} \tau \approx 0$

Figure 14. Vertical profiles of the wave magnitude $A$ (left panel), phase $\phi$ (center panel), and mean flow (right panel) at three times in two layers. The parameter values are $n_{1} / k=\sqrt{2}, N_{2} / N_{1}=2$, $\epsilon=0.025$, and $\alpha=0.1$. The line conventions are the same as Fig. 3 .

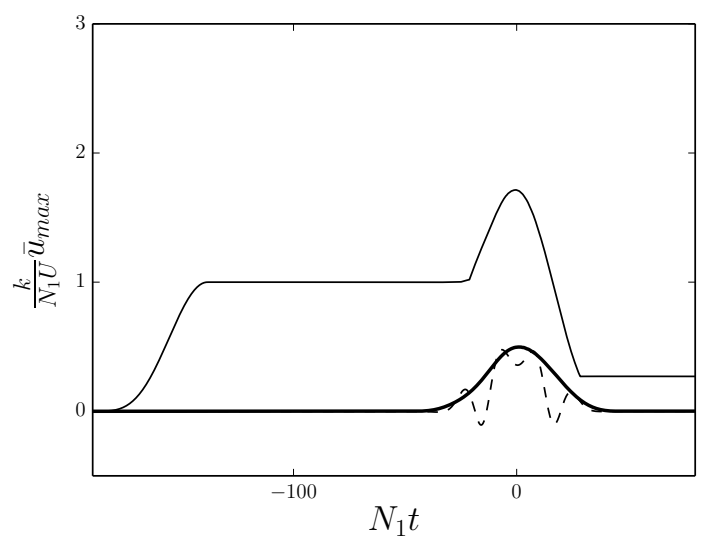

Figure 15. Time history of the maximum of the mean flow. The dashed line is the velocity jump at the interface while the thick solid line that is the maximum the interference mean $\bar{u}_{\mathrm{i}}$. The parameter values are $n_{1} / k=1 / \sqrt{2}, N_{2} / N_{1}=2, \epsilon=0.025$, and $\alpha=0.1$.

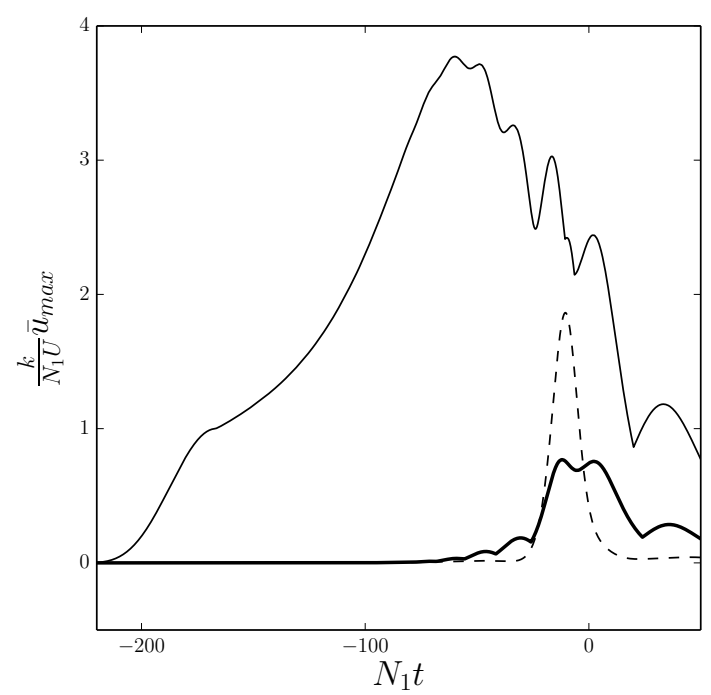

Figure 16. Time history of the maximum of the mean flow. The dashed line is the velocity jump at the interface while the thick solid line that is the maximum the interference mean $\bar{u}_{\mathrm{i}}$. The parameter values are $n_{1} / k=0.4, N_{2} / N_{1}=2, \epsilon=0.025$, and $\alpha=0.1$.

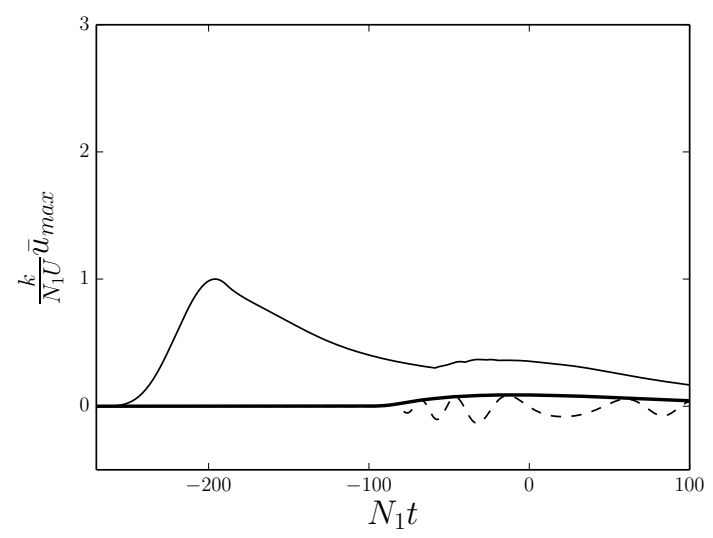

Figure 17. Time history of the maximum of the mean flow. The dashed line is the velocity jump at the interface while the thick solid line that is the maximum the interference mean $\bar{u}_{\mathrm{i}}$. The parameter values are $n_{1} / k=\sqrt{2}, N_{2} / N_{1}=2, \epsilon=0.025$, and $\alpha=0.1$.

\section{Conclusions}

Atmospheric observations indicate that the tropopause altitude is more likely to experience turbulence and large amplitude waves than other altitudes. The abrupt change in the buoyancy frequency suggests that such observations are related to the dynamics of internal waves near the tropopause. Previous numerical simulations conclude that internal waves will create a wave-induced jet-like mean flow in the tropopause vicinity that is likely responsible for at least some of the observations. An idealized low-dimensional model of such waves is treated here. The model consists of three coupled nonlinear Schrodinger equations along with linear interfacial conditions. 


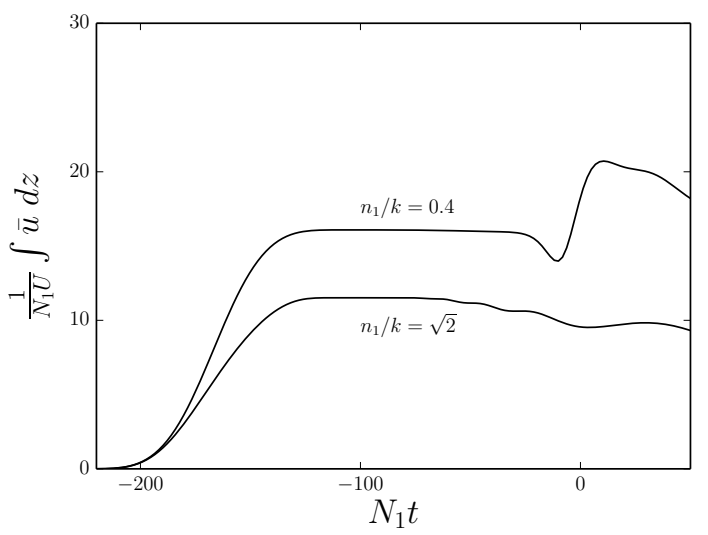

Figure 18. Temporal evolution of the vertical integral of the mean velocity for $n_{1} / k=0.4$ and $n_{1} / k=\sqrt{2}$.

Numerical solutions with weak dispersion $\left(n_{1} / k=1 / \sqrt{2}\right)$ show that the wave-induced mean flow is greatest near and under the interface, similar to previous full numerical simulations by McHugh (2008). Furthermore, this mean flow is discontinuous at the interface and would be a region with enhanced shear in a viscous flow. The previous full numerical results did not allow the velocity to be discontinuous at the interface, and this could have impacted the final results in that study.

However, the results here also show that dispersion may dominate the motion and can act to greatly enhance the jetlike flow or weaken it, depending on the value of $n_{1} / k$. With $n_{1} / k<1 / \sqrt{2}$, the jet-like mean flows are strengthened and they can happen at any altitude, not necessarily near the interface. With $n_{1} / k>1 / \sqrt{2}$, dispersion tends to smooth the localized effects near the interface.

The mean flow found here also has the oscillatory interference component. The results show that these mean-flow oscillations are transferred to the wave envelopes and can become exaggerated in the tail of the incident wave packet when $n_{1} / k<1 / \sqrt{2}$. Furthermore, the vertical wavelength of the oscillation decreases during the period where incident and reflected waves overlap. These are nonlinear features of the waves that do not occur in linear theory. Note that this feature of the mean flow indicates that the waves create a structure that is wider horizontally than the incident waves but shorter in the vertical. Hence there is a cascade of energy to "flatter" structures.

The observations of McHugh et al. (2008a) showed layers at the tropopause region with large values of vertical velocity. The terrain was dominated by a single large peak (Mauna Kea), and thus these structures were likely caused by mountain waves. There are many similarities between those experimental results and the present model results; most notably, the mean flow oscillations in the model are similar to the layer structure in the experiments. However, differences are also significant; for example, the experiments show large ver-
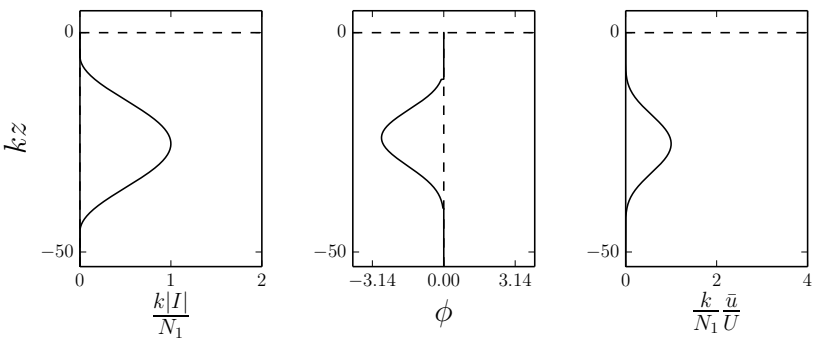

(a) $N_{1} \tau \approx-65$
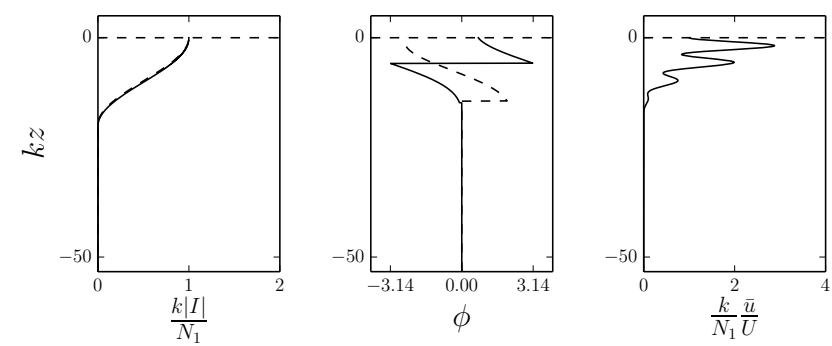

(b) $N_{1} \tau \approx 0$
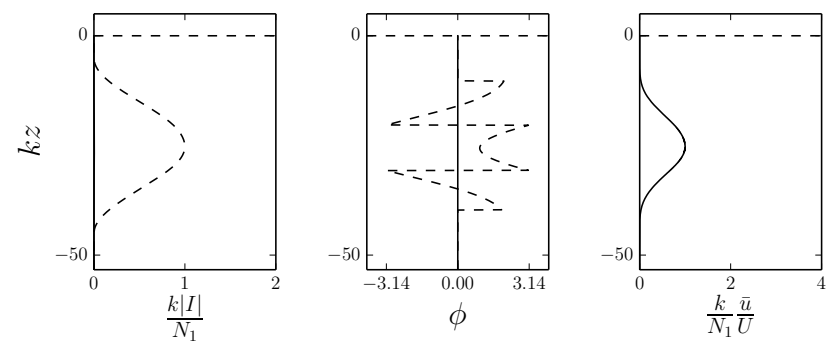

(c) $N_{1} \tau \approx+65$

Figure 19. Vertical profiles of the wave magnitude (left panel), phase (center panel), and mean flow (right panel) at three time values with a rigid lid, with $n_{1} / k=1 / \sqrt{2}, \epsilon=0.025$, and $\alpha=0.1$. The line conventions are the same as Fig. 3.

tical velocity whereas the model finds large horizontal velocity. A detailed comparison is difficult since the experiments treated mountain waves, a much more complex wave system. Hence it is tempting but premature to connect the mean flow oscillations found here to the structures observed in McHugh et al. (2008a).

Acknowledgements. Funding provided by the Physical Mathematics Program at the Air Force Office of Scientific Research.

Edited by: R. Grimshaw

Reviewed by: three anonymous referees 


\section{References}

Ames, W. F.: Numerical methods for partial differential equations, Academic Press, New York, NY, USA, 1977.

Andrews, D. G. and McIntyre, M. E.: An exact theory of nonlinear waves on a Lagangian-mean flow, J. Fluid Mech., 89, 609-646, 1978a.

Andrews, D. G. and McIntyre, M. E.: On wave action and it's relatives, J. Fluid Mech., 89, 647-664, 1978b.

Born, M. and Wolf, E.: Principles of Optics, Pergamon, Cambridge, UK, 1959.

Dosser, H. V. and Sutherland, B. R.: Weakly nonlinear nonBoussinesq internal gravity wavepackets, Physica D, 240, 346356. 2011.

Griffiths, S. D., Grimshaw, R. H. J., and Khusnutdinova, K. R.: Modulational instability of two pairs of counter-propagating waves and energy exchange in a two-component system, Physica D, 214, 1-24, 2006.

Grimshaw, R. H. J.: Nonlinear internal gravity waves and their interaction with the mean wind, J. Atmos. Sci., 32, 1779-1793, 1975.

Grimshaw, R. H. J.: The modulation and stability of an internal gravity wave, Memoires Societe Royale des Sciences de Liege, 299-314, 1976.

Grimshaw, R. H. J.: Mean flows induced by internal gravity wave packets propagating in a shear flow, J. Fluid Mech., 90, 161-178, 1979.

Grimshaw, R. H. J. and McHugh, J. P.: Steady and unsteady nonlinear internal waves incident on an interface, Q. J. Roy. Met. Soc., 139, 1990-1996, 2013.

Knobloch, E. and Gibbon, J. D.: Coupled NLS equations for counter propagating waves in systems with reflection symmetry, Phys. Lett. A, 154, 353-356, 1991.

McHugh, J. P.: Mean flow generated by an internal wave packet impinging on the interface between two layers of fluid with continuous density, Theor. Comp. Fluid Dynam., 22, 107-123, 2008.

McHugh, J. P.: Internal waves at an interface between two layers of differing stability, J. Atmos. Sci., 66, 1845-1855, 2009.

McHugh, J. P., Dors, I., Jumper, G. Y., Roadcap, J., and Murphy, E. and Hahn, D.: Large variations in balloon ascent rate over Hawaii, J. Geophys. Res., 113, D15123, doi:10.1029/2007JD009458, 2008a.
McHugh, J. P., Jumper, G. Y., and Chun, M.: Balloon thermosonde measurements over Mauna Kea, and comparison with seeing measurements, Pub. Astro. Soc. Pac., 120, 1318-1324, 2008 b.

Partl, W.: Clear air turbulence at the tropopause levels, Navigation, 9, 288-295, 1962.

Scorer, R. S.: Theory of waves in the lee of mountains, Q. J. Roy. Meteorol. Soc., 75, 41-56, 1949.

Shrira, V. I.: On the propagation of a three-dimensional packet of weakly non-linear internal gravity waves, Int. J. Non-Lin. Mech., 16, 129-138, 1981.

Smith, R. B., Woods, B. K., Jensen, J., Cooper, W. A., Doyle, J. D., Jiang, Q., and Grubisic, V.: Mountain waves entering the stratosphere, J. Atmos. Sci., 65, 2543-2562, 2008.

Sutherland, B. R.: Finite-amplitude internal wavepacket dispersion and breaking, J. Fluid Mech., 429, 343-380, 2001.

Sutherland, B. R.: Weakly nonlinear internal gravity wavepackets, J. Fluid Mech., 569, 249-258, 2006.

Tabaei, A. and Akylas, T. R.: Resonant long-short wave interactions in an unbounded rotating stratified fluid, Stud. Appl. Math., 119, 271-296, 2007.

Tabaei, A., Akylas, T. R., and Lamb, K. G.: Nonlinear effects in reflecting and colliding internal wave beams, J. Fluid Mech., 526, 217-243, 2005.

Thorpe, S. A.: On the shape of progressive internal waves, Philos. T. Roy. Soc. A, 263, 563-614, 1968.

Thorpe, S. A.: On the reflection of a train of finite-amplitude internal waves from a uniform slope, J. Fluid Mech., 178, 279-302, 1987.

Voronovich, A. G.: On the propagation of a packet of weakly nonlinear internal waves in a medium with constant Vaisala frequency, Izv. Atmos. Ocean Phys., 18, 247-250, 1982.

Wolff, J. K. and Sharman, R. D.: Climatology of upper-level turbulence over the contiguous United States, J. Appl. Meteorol. Clim., 47, 2198-2214, 2008.

Worthington, R. M.: Tropopausal turbulence caused by the breaking of mountain waves, J. Atmos. Sol.-Terr. Phy., 60, 1543-1547, 1998. 